\title{
PUBLICATION PRODUCTIVITY OF CZECH SOCIOLOGY IN SCIENTIFIC JOURNALS WITHIN THE LAST DECADE
}

\author{
Josef Basl \\ Daniel Münich \\ Oleg Sidorkin
}
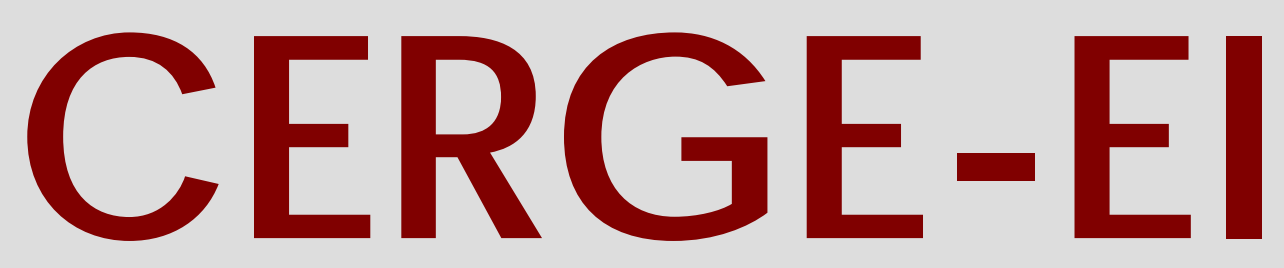

Charles University Centerfor Economic Research and Graduate Education Academy of Sciences of the Czech Republic Ec onomic s Institute 


\title{
Working Paper Series (ISSN 1211-3298)
}

\section{Publication Productivity of Czech Sociology in Scientific Journals Within the Last Decade}

\author{
Josef Bas1 \\ Daniel Münich \\ Oleg Sidorkin
}

CERGE-EI

Prague, September 2009
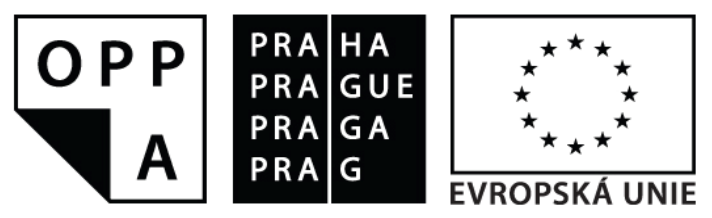

EVROPSKÝ SOCIÁLNÍ FOND

PRAHA \& EU: INVESTUJEME DO VAŠÍ BUDOUCNOSTI

Projekt je financován Evropským sociálním fondem, rozpočtem hl. města Prahy a státním rozpočtem 
ISBN 978-80-7343-194-5 (Univerzita Karlova. Centrum pro ekonomický výzkum a doktorské studium)

ISBN 978-80-7344-183-8 (Národohospodářský ústav AV ČR, v.v.i.) 


\title{
Publication Productivity of Czech Sociology in Scientific Journals Within the Last Decade*
}

\author{
Josef Basl ${ }^{\dagger}$ \\ Institute of Sociology, \\ Academy of Sciences of the \\ Czech Republic
}

\author{
Daniel Münich Oleg Sidorkin
}

CERGE-EI $I^{\dagger}$

\begin{abstract}
The paper maps publication outcomes of Czech sociologists in journals with impact factor within the last decade. The methodology we employ is similar to that used in recently published work analyzing the productivity of Czech economists. Our work is based on data from the Web of Science and covers the 1998 to 2007 period. Publication outcomes for sociology are presented within the context of the whole field of social sciences. The productivity of individual authors is based on data about published articles, impact factors of journals, and co-author shares and citations. Our analysis does not provide detailed insight on publications in another most important publication channel - scientific monographs - due to non-existence of suitable quality indicators. Our analysis shows that similar authors rank among the first fifty using the selected indicators of impact factor and citation indexes. The order differs fairly strongly according to the indicator selected, however. It turns out that publication-wise, there are rather strongly differentiated groups of sociologists also in the Czech Republic.
\end{abstract}

Key words: sociology, impact factor, citation, Czech Republic, articles, research JEL classification: A10, A12, I2

\footnotetext{
* Our thanks to the heads of the participating sociology departments, which kindly provided the necessary information. We also thank our assistants, Olga Popová and Anna Tůmová for their assistance with data preparation.

$\uparrow$ Direct all correspondence to: e-mail: josef.basl@soc.cas.cz or e-mail: daniel.munich@cergeei.cz.

* CERGE-EI is a joint workplace of the Center for Economic Research and Graduate Education, Charles University, and the Economics Institute of Academy of Sciences of the Czech Republic. Address: CERGE-EI, P.O. Box 882, Politických vězňů 7, Prague 1, 111 21, Czech Republic
} 


\begin{abstract}
Abstrakt
Článek mapuje publikační výsledky českých sociologů v impaktovaných odborných časopisech v posledních deseti letech. Práce vychází z údajů Web of Science. Metodologicky navazuje na nedávno publikovanou analýzu pro oblast české ekonomie. Publikační výsledky v oblasti sociologie jsou zasazeny do rámce situace v celé oblasti společenských věd. Na základě bibliometrických údajů o počtech publikovaných článků, impakt faktorech časopisů, autorských podílech a citacích jsou prezentovány výsledky jednotlivých autorů. Studie neposkytuje vhled do publikační produkce $\mathrm{v}$ dalším významném segment - vědeckých monografiích - protože pro ně dosud nejsou k dispozici použitelné ukazatele kvality. Analýza ukazuje, že mezi nejlepšími padesáti sociology se většina vyskytuje bez ohledu na zvolený ukazatel založený na impakt faktorech nebo citacích. Jejich pořadí se však v závislosti na zvoleném indikátoru nezř́íka výrazně liší. Ukazuje se, že i v České republice existují různé skupiny sociologů podle formy publikování.
\end{abstract}




\section{Introduction}

In developed countries, the evaluation of the volume and above all the quality of basic research outcomes has been common for decades (Fox 1983; Morley, Ferrucci 2008). This has also been evidenced in the development of scientometrics as an independent academic discipline (Kademani, Kalyane 1996). It has been developing rapidly not only due to computer and internet tools development, the primary stimulus has been the increased interest in research and development (R\&D) quality as factors of technological, social and economic growth. The effort to evaluate R\&D results is an inseparable part of governmental endeavors to setup basic research systems appropriately and to manage them efficiently. In the Czech Republic, the issues around basic research productivity evaluation are increasingly relevant due to the R\&D funding reform being implemented. Self-reflection within academic disciplines from the viewpoint of acquired knowledge and also organizational and individual performance will undoubtedly begin to fill the gap in $R \& D$ results evaluation, including international comparisons of Czech academic disciplines and sub-disciplines. This is especially true for social sciences and humanities, which strive for full integration into international contexts after decades of isolation from international development. Information provided on the qualitative performance of Czech academic disciplines and subdisciplines in the 'Analysis of the existing state of research, development and innovation in the Czech Republic and a comparison with the situation abroad in 2008' (2008), the key annual publication of the Office of the Government of the Czech Republic, is very incomplete. So far, a more detailed overview of the current social science field productivity only exists for Czech economics (Gregor, Schneider 2005; Turnovec 2005; Münich 2006; Macháček, Kolcunová 2008).

In this article, we present an insight into the quantity and quality of academic article as reflected by impact factors of journals presenting basic research results authored or coauthored by academic sociologists working in the Czech Republic. For many reasons mentioned further on, we focus on the period 1998 to 2007. We work with articles published in journals carrying an impact factor in the Thomson Reuters Web of Science database. Publication performance evaluation methodology directly follows upon Münich's overview for the field of economics (2006). Our qualitative assessment is 
based on impact factor (hereafter referred to as $\mathrm{IF}^{2}$ ) values of journals featuring the authors' publications and on the citation response to their articles and other works.

We are well aware that scientific production is not limited to academic articles in journals with IF, especially so in humanities and partly in social sciences as well. Our ambition and aim, however, is not to comprehensively evaluate the production by Czech sociologists. This should be done by the government. Our analysis serves as a first and partial view of the skills of Czech sociologists to enter the space where sociologists in advanced countries compete, where bibliometric data are commonly used as one of the scientific productivity evaluation criteria in basic research.

In our analysis, we use data on the IF of journals where the authors have published their articles. Articles in journals with IF have undergone the journals' tough review process and it is precisely the number of citations of an article from a certain journal that establishes the journal's IF. This is the main reason why IF is deemed to be a fairly reliable quality indicator for the given journal. Journals with higher response in the academic community very often exhibit higher IF levels. IF is of course not an ideal quality measure for every single article published in the journal. Nevertheless, it is probably the best metric meeting these fundamental criteria: it is fairly easily accessible, i.e. verifiable; it is independent of the analyzer's subjective perception; it reflects the international scientific community response to the articles in the journal; and it is resistant to manipulation by individual authors.

Citation indexes represent an alternative to IF. However, we consider them to be less useful quality indicators than IF for our purposes as citations are manipulable through self-citation or coordinated mutual citing among multiple authors. A simple citation total also fails to account for citing source quality and the reasons for citing. Besides, citations accumulate for years following the article publication, so they often cannot enter into the current situation evaluation.

\footnotetext{
${ }^{2}$ According to the Web of Science, a journal's IF reflects the number of citations appearing in the Journal Citation Reports (JCR) in a given year of articles which have been published in a given journal over past two years. An impact factor of 2 means that, for example, articles published one or two years ago in a given journal have been cited two times during the year 2006 .
} 


\section{International context}

Our insight into the Czech sociologists' publication productivity needs to be perceived in the context of the entire social science and humanities academic sphere in the Czech Republic. Therefore, we include a basic comparison of the volume of scientific production in social science/humanities academic journals for European countries of similar size (the Netherlands, Sweden, Austria) without the communist dictatorship heritage, of similar size and with English as a non-native language. The comparison in Table 1 shows the number of scientific articles in journals with IF in 2006 and their citation totals.

The Czech Republic's publication production in social sciences and humanities in journals with IF is extremely low when compared internationally. Moreover, the majority of articles are published in local Czech-Slovak journals. The low average number of citations also includes self-citations and local citations and their proportion is relatively high in the Czech Republic. The international academic response to the results of Czech social sciences and humanities is apparently very low.

Figure 1 shows the distribution of articles in journal decile groups, where the highest group represents $10 \%$ journals with the highest IF in the given field. Column height for the Czech Republic shows article totals ${ }^{3}$ and the results for other countries are weighted in inverse proportionality to the country's population so that the results are not influenced by certain differences in population.

Apparently, the overall publication productivity in the Czech Republic fails by far to reach either the quality or the volume of the countries included in our comparison. Whereas the production gap in natural science disciplines is apparent in the higher quality half of journals (5th to 10th decile), the production gap in social sciences and humanities is much wider in the entire journal spectrum. It needs to be kept in mind that

\footnotetext{
${ }^{3}$ Relevant data were found in the electronic Web of Science database, a part of the Thomson Reuters ISI Web of Knowledge (http://www.isiknowledge.com) electronic information resource. Apart from Web of Science, Journal Citation Reports (where journal IF can be searched) or Current Contents tools are also available.
} 
the stated data do not take into account national specificities such as the number of researchers in the field, spending per academic discipline, or the intellectual dispositions or academic working conditions.

It is almost exclusively economists, psychologists and sociologists who publish in the framework of Czech SSH, as clearly evidenced in Table 2. It should be noted that most articles in these disciplines are also published in local Czech-Slovak journals (total 8 journals) and most of them are in Czech or Slovak. The limited number of potential readers predetermines the low international academic response, as measured by citations. A more detailed insight into the main social sciences and humanities is provided in Figure 2, which, like Figure 1, shows article distribution in journals ordered by IF within the discipline. High columns in the low IF area correspond to CzechSlovak journals with IF.

\section{Data and methods}

Our publication productivity overview for the field of sociology in the Czech Republic is based on the data available in the ISI Web of Knowledge database. The 2007 IF values for individual journals have been taken from the Thomson Reuters Journal Citation Reports database.

Since our aim was to map out a publication overview in the area of sociology, the first step was to identify organizations and departments addressing sociology issues in the Czech Republic and then individuals dealing with sociological issues academically. Based on information from relevant departmental websites, we created a primary list of authors. We then contacted the heads of these departments via email and asked them for basic information. ${ }^{4}$ We contacted a total of 16 workplaces $^{5}$ (for details, see Table A3).

\footnotetext{
${ }^{4}$ We asked them to complete a list of their colleagues according to the current situation (including a potential note of which colleagues do not deal with sociology) and to add individual workers' year of birth.

${ }^{5}$ With the exception of the Institute of Sociology of the Academy of Sciences of the Czech Republic, as this is the workplace of one of the co-authors and the sociology affiliation did not need to be verified here.
} 
We searched the ISI Web of Knowledge database for articles by the listed authors. ${ }^{6}$ In the course of the search, co-authors were added to the list, if relevant as regards the topic of the article. Since the aim of this study is to describe a recent period, not the entire long history of sociology in the Czech Republic, we only focused on articles published between 1998 and 2007. ${ }^{7}$ We limited our analysis to the 'Article' type of publication. ${ }^{8}$ We checked the affiliation of authors with an organization based in the Czech Republic. ${ }^{9}$ We also observed the source journal subject category for the articles. Based on this database of articles by Czech authors, we identified a list of source journals where the authors published (see Table A1) and an overview of relevant subject categories (see Table A2).

Sociology overlaps with other disciplines, we therefore distinguished a narrower and a broader group of sociology journals. The subject category of sociology journals in the narrower sense explicitly contains the word 'sociology' in WoS. ${ }^{10}$ Sociology journals in the broader sense cover all journals where the searched articles were published, i.e. including the narrow group of journals. This distinction corresponds inter alia to the dual possible relation of individual authors to the field of sociology: either they identify with the field itself or with an organization addressing sociology issues.

In the next step, we attributed the articles with the IF value of the journal ${ }^{11}$ where they were published, and we also accounted for author shares. ${ }^{12}$ In case of co-authorship, each author received an equal share in the journal IF. Then, the sum of IF achieved for all published articles was calculated for each author. The complete overview of articles

\footnotetext{
${ }^{6}$ We only searched partial databases in the Web of Knowledge: Social Sciences Citation Index (SSCI) and Arts and Humanities Citation Index (AHCI).

${ }^{7}$ The initial year was chosen due to the connection of this article to the method used in drafting the publication performance overview for economics (Münich 2006) and also with regards to the fact that the main local sociologically profiled journal (Sociologický časopis / Czech Sociological Review) has had IF in the Journal Citation Reports database since 2001.

${ }^{8}$ Several articles, displaying the feature of a yearbook, were not included in further processing (e.g. under names like 'Czech Republic' or 'Political Data Yearbook - Czech Republic'). An abstract was not included in them, either. Therefore, we do not consider them full articles.

${ }^{9}$ In line with the Web of Science search rules, author's names were entered in the form 'surname', 'space', 'first letter of first name' (without diacritics).

${ }^{10}$ Specifically, these were articles from the journals numbered 1 - 15 (for more details, see Table A1).

${ }^{11}$ With few exceptions, we worked with 2007 IF values, as stated in Table A1.

${ }^{12}$ For articles with e.g. four co-authors, we worked with author share IF/4 for each of the authors.
} 
ordered by author is included in Table A5 (if an article had more authors, it is included more times).

We established each author's citation total using the WoS Cited Reference Search tool with the same time and publication type limits as in article search.

\section{Results}

242 articles were found in the WoS for the 1998-2007 period, i.e. 314 records, taking account of articles with multiple authors, (see Table A5). There were 108 article authors. 200 articles fall in the category of narrowly focused sociology journals ${ }^{13}$, out of which the total of 170 were published in the Sociologický časopis / Czech Sociological Review. Out of all articles, 157 were written in Czech, 15 in Slovak, 62 in English and 8 in another foreign language (German, French, Russian).

Table 3 shows a basic results overview. Columns (1) - (4) contain individual authors' IF totals achieved by article publication, while columns $\left(1^{\circ}\right)-\left(4^{\circ}\right)$ contain the corresponding order of authors. The order of authors (column 1') is based on the IF achieved in the narrow group of sociology journals in a world language (column 1). Other columns (2) - (4) state the achieved IF totals for other combinations of journal groups and languages. In Table 3, the results for a certain combination of journals and article language only take into consideration authors with non-zero IF value. This shows that groups of Czech sociologists are fairly strongly divided either according to the language they publish in or the thematic breadth of their sociological research.

The number of published articles for 1998 to 2007 is of course influenced by the length of time the author has been active in the field. Shorter time applies primarily to younger sociologists who only began their academic career after 1998. Therefore, in Table 4, we compare authors under 40 years of age (born after 1967). The order in the top positions does not differ significantly for different alternatives, however, further down we again

\footnotetext{
${ }^{13}$ These are journals under numbers 1 to 15 in Table A1.
} 
encounter relatively large differences. It is obvious that many younger sociologists have successfully published in world languages in sociology journals with broader focus.

Czech sociologists publish predominantly in the Sociologický časopis / Czech Sociological Review (SČ). It is the only Czech journal with IF included in the international ISI Web of Knowledge database and it falls into the group of sociology journals in the narrower sense. Of the total of articles published in journals with IF, 70 percent were published in this journal. Another 5 percent were published in the Slovak journal with IF called Sociológia (S). Only 25 percent of articles were then published in truly international journals - where a larger proportion of authors come from different countries. This is a very low share given the fact that WoS contains almost 100 sociology journals in the narrow sense and many more journals in the broader sense. ${ }^{14}$ As the SČ is publishing articles both in Czech and English language - four and two issues per year, respectively - a simple look at the most cited articles influencing the IF of the journal the most has shown that articles from both editions, Czech and English, has contributed about the same extent to the total IF.

The high concentration of articles by the Czech sociological community in the only local journal is striking. It is true that social science and namely humanities journals have a significantly stronger tendency for local thematic orientation than natural science journals (Moed 2005) but the share of articles published in SČ seems exceptionally high, nevertheless. As the majority of articles published in SČ are written in Czech, the question then arises to what extent the Czech sociology community focuses on issues that cannot contribute to the discipline's international knowledge to any greater degree, and to what extent it is the result of low ambitions or dispositions in the academic community to publish their results in a format designed for the international community.

\section{Citation analysis}

Citation analysis and its indexes represent the standard bibliometric alternative to IF. Whereas IF derives the potential impact of a specific article from the response to an average article in the journal for a certain period of time, citation indexes are directly linked to specific articles by authors. This of course represents an advantage. On the

\footnotetext{
${ }^{14}$ Several of the 100 journals are focused on other countries, however.
} 
other hand, citation indexes have many fundamental disadvantages for our kind of evaluation, which we discuss in the opening section and which need to be taken into account when interpreting the results presented here.

Table 5 shows a whole range of citation indexes for our list of authors. The Hirsch index (h-index) is an often used citation index and its 1998-2007 values are stated in column (3). ${ }^{15}$ The author's H-index (value from WoS) in our case equals the order of the article (by the given author), whose number of citations equals or exceeds the order of this article. Articles are listed in descending order according to citations. An obvious weakness of h-index in the Czech case is the low range of values between 0 and 3. By way of comparison let's state that the world renown sociologist Zygmunt Bauman reaches h-index 5 for a comparable period. Among four Czech authors with the highest h-index (value 3), two (P.Matějů and K.Vlachová) rank among the first ten according to IF (Table 3). Of the other two, T.Kostelecký achieves high h-index despite the fact that he does not have articles in world languages in IF journals. J.Řehák's high h-index is probably caused by the focus of his articles on statistical data analysis methods, which entails frequent citations by method users. Among 14 other sociologists with h-index 2, just two (Z.Vajdová and J.Buriánek) are not included in Table 3 ordered by the achieved IF.

Other columns in Table 5 show a whole range of alternative citation indexes. Panel A contains citation indexes based on the authors' articles from the period we explore 1998 to 2007.

Tables 3 and 5 (panel A) rank many important Czech sociologists low according to IF as well as citation indexes. This is mainly because many of them published their most important works before 1998. This corresponds to the results in panel B, which are not limited by a time period. There is of course a great difference between measuring citation response for an author's life-work and for work published in a certain period.

\footnotetext{
${ }^{15} \mathrm{H}$-index (Hirsch index) expresses the quantitative evaluation of the quality of articles published by a given person. It equals the order of publication (publications are listed in descending order according to number of citations) the number of citations of which equals or exceeds its order. Like IF, h-index does not address comparing performance in different fields, it is bound to a given academic field and the results are difficult to compare between fields.

(Source: http://knihovny.cvut.cz/en/veda/h-index.html)
} 
The data in column (13) of Table 5 take into account the citations of our authors' monographs, if cited in journals registered in WoS. This indicator identifies significant Czech sociologists much better.

The fact stated above that comparisons based on IF by definition do not capture publications in academic monographs plays an important role in our comparison. Moed (2005) states on this issue that publication in social science/humanities research happens in different, partly overlapping worlds of international journals, monographs, local journals and other printed media. In this context, he claims that the importance of journals as a communication medium, as expressed by the share of references to papers published in journals to the overall number of citations, for sociology is 37 percent of the worldwide average and the share of references to papers in journals registered in WoS to the overall number of references from journals is 74 percent. The precise shares for the Czech Republic are not known but the role of monographs in the Czech Republic is probably significantly higher than Moed states (2005). Information on the number of citations of monographs in other monographs for the Czech Republic unfortunately does not exist yet. Extended citation analysis is fairly demanding and in other countries, it is usually carried out by a specialized organization. Such an organization does not exist yet in the Czech Republic.

More as a supplement, Table 6 includes information about the most frequently cited articles published in the period 1998-2007 (cited at least five time in total). Article titles can be found in Table A4. Among the authors of most cited articles, K. Vlachová has written 3, and P. Matějů and J. Večerník two each. As the number of citations does not take into account the potentially different quality and significance of citing sources, we also include the totals without self-citations and citation totals from international sources. The values of the last data emphasize the low international reflection of Czech sociology results, as evidenced by our entire analysis.

\section{Conclusion}

The aim of this article is to offer a partial insight into the publication performance in the field of sociology in the Czech Republic and thus to contribute to the self-reflection 
within this academic discipline. Results are intentionally set in an international context, as it is highly relevant in the area of basic research.

Our analysis shows that similar authors rank among the first fifty using the selected indicators of IF and citation indexes. Within the first fifty positions, the order differs fairly strongly according to the indicator selected, however. It turns out that publicationwise, there are rather strongly differentiated groups of sociologists. Although citation indexes themselves generate rankings with fairly high positive mutual correlations, we also encounter many variations. This is also the consequence of fairly significant differences in the authors' publication practices. The differences show that bibliometric indicators need to be chosen with care based on the true purpose of comparing.

We are aware of the fact that any evaluation of academic outcomes such as this one is to an extent arbitrary and has real limitations. As our analysis focuses primarily on academic journals with IF, the main apparent shortcoming of our analysis is the fact that it accounts insufficiently for basic research results spread in the form of non-IF refereed journals and academic monographs. Although sociology is among the social science disciplines where new findings are often spread in the form of articles (Moed) and it even features the third highest number ${ }^{16}$ of academic journals with IF after economics and psychology, the important role of academic monographs in the field is obvious. To capture the contribution of monographs to academic knowledge is, however, much more demanding technically and for the time being unfeasible in the Czech Republic. The quality of book publications can be bibliometrically grasped using the so-called extended citation analysis. To carry out such an analysis is, however, extremely demanding due to the necessary background in the form of very large citing and cited indexes. A full-blown extended citation analysis can only be carried out in the framework of a large and long-term international project. As currently no such project enabling comparison including the Czech Republic exists, we present the results of at least partially extended citation analysis. In the framework of citations in IF journals, it also takes into consideration citations referring to the author's work published in the form of academic monographs, i.e. not just academic articles. Based on the overview

\footnotetext{
${ }^{16} \mathrm{WoS}$ registers almost 100 journals in the Sociology category. A great number of other journals, where many sociologists publish, can be considered thematically close.
} 
gained from such an analysis we may believe, however, that an extended citation analysis would not have altered our findings in a substantial way either. This is given by the fact that an absolute majority of sociology monographs by Czech authors published in 1998 to 2007 were not published in a world language, meaning they could not reach the international academic community to a considerable degree and thus significantly contribute to the development of knowledge in a discipline of a largely international character.

Results of individual authors are undoubtedly influenced by a number of specific factors such as e.g. the scope of their thematic range, relation of their research focus to issues of a more "local" or "global" character, the author's publication strategy (fewer articles with one author, more articles based on co-authorship), the length of academic career (younger researches, international stays, executive roles, political career), the amount of work load devoted to scientific work, and the type of academic career as sociologists based at universities usually do a lot of teaching in addition to their research. Although analyses of the influence of these factors exist internationally (Fox 2005), it would be very hard in our case to quantify it precisely and without subjective judgments. Therefore, we do not address these issues in the text and we let the IF and citation indexes speak, which are independent of subjective feelings.

When evaluating results in the area of Czech social sciences and humanities, we must not forget the significant negative impact of ideological biases and interventions of the communist dictatorship on the intellectual background in social sciences and humanities. A question remains about the extent to which the relatively low contribution of current Czech sociology to the international development in the field is still a consequence of historical circumstances and to what extent it is caused by the absence of R\&D outcome evaluation in the Czech Republic in general and the resulting weak competition motivation within the academic community.

\section{Information about the authors}

Josef Basl is a doctoral student and a research fellow at the Sociology of Education and Stratification Department at the Institute of Sociology of the Academy of Sciences of 
the Czech Republic. He focuses primarily on the issues of educational inequalities and educational policy.

Daniel Münich is an independent research worker and a university teacher at CERGEEI, a joint workplace of Charles University and the Academy of Sciences of the Czech Republic. He focuses primarily on issues of educational economy, labor market economy and the evaluation of academic outcomes.

Oleg Sidorkin is a doctoral student at CERGE-EI, a joint workplace of Charles University and the Academy of Sciences of the Czech Republic. He focuses primarily on issues of academic functioning. 


\section{Bibliography}

Analysis of the existing state of research, development and innovation in the Czech Republic and a comparison with the situation abroad in 2008, (2008). Office of the Government of the Czech Republic, Research development Council. At www: http://www.vyzkum.cz/FrontClanek.aspx?idsekce=513444

Fox, M.F. 1983. "Publication Productivity among Scientists: A Critical Review“. Social Studies of Science, 13 (2): 285-305.

Fox, M.F. 2005. "Gender, Family Characteristics, and Publication Productivity among Scientists“. Social Studies of Science, 35 (1): 131-150.

Gregor, M., O. Schneider. 2005. "The World is Watching: Rankings of Czech and Slovak Economics Departments. Finance a úvěr - Czech Journal of Economics and Finance, 55 (11-12): 518-530.

Jurajda, Š. 2008. “Česká věda potřebuje nezávislý audit". Lidové noviny, 6.5.2008, s 11.

Kademani, B. S., V.L. Kalyane. 1996. "Bibliometric indicators for publication productivity analysis of an individual scientist“. JISSI : the international journal of scientometrics and informetrics 2(4): 49-58.

Macháček, M., E. Kolcunová. 2008. "Hirsch index and rankings of Czech economists“". Politická ekonomie, 56 (2): 229-241.

Moed, H. F. 2005. Citation Analysis in Research Evaluation, (vol. 9). London: Kluwer.

Morley, J.E., L. Ferrucci. 2008. "Publication Productivity in Geriatrics: 1995-2006“. Journal of Gerontology: Medical Sciences, 63 (6): 584-585.

Münich, D. 2007. "Extraliga české akademické ekonomie“ (online). iHNed, 31.5. 2007. Dostupný z www: http://ihned.cz (cit. 5.6. 2008)

Münich, D. 2006. "Recent Publication Productivity of Czech Economists". Finance a úvěr - Czech Journal of Economics and Finance, 56 (11-12): 522-533.

Turnovec, F. 2005. “Institucionální vědecký kapitál a individuální výkonnost ekonomů v ČR“. Finance a úvěr - Czech Journal of Economics and Finance, 55 (11-12): 531545. 


\section{Tables and figures}

Table 1. Population of countries, number of articles in IF journals produced by authors with an affiliation in a given country, share of articles published in journals issued in a given country in the field of social sciences and humanities, average number of citations per article. Data for 2006.

\begin{tabular}{|l|c|c|c|c|}
\hline \multicolumn{1}{|c|}{ Country } & $\begin{array}{c}\text { Population } \\
\text { in mil. }\end{array}$ & $\begin{array}{c}\text { Number of } \\
\text { articles }\end{array}$ & $\begin{array}{c}\text { Share in } \\
\text { national } \\
\text { journals }\end{array}$ & $\begin{array}{c}\text { Average } \\
\text { number of } \\
\text { citations per } \\
\text { article }\end{array}$ \\
\hline \multicolumn{5}{|c|}{ Sciences (natural science) } \\
\hline $\begin{array}{l}\text { Czech } \\
\text { Republic }\end{array}$ & 10.27 & 5819 & 2.31 \\
\hline Netherlands & 16.34 & 19546 & & 4.04 \\
\hline Sweden & 9.08 & 14991 & & 3.62 \\
\hline Austria & 8.28 & 7712 & & 0.42 \\
\hline \multicolumn{5}{|c|}{ Social sciences and humanities } \\
\hline Rechublic & 10.27 & 194 & 0.71 & 1.63 \\
\hline Netherlands & 16.34 & 2540 & $*$ & 1.32 \\
\hline Sweden & 9.08 & 1265 & 0.01 & 1.46 \\
\hline Austria & 8.28 & 355 & 0.07 & \\
\hline
\end{tabular}

* Data for Netherlands not mentioned as the publisher Elsevier is based in this country and therefore it is not possible to distinguish between domestic and foreign journals. 
Figure 1. Number of articles in IF journals published in 2006 by authors with an affiliation in a given country (weighted on population of the Czech Republic)
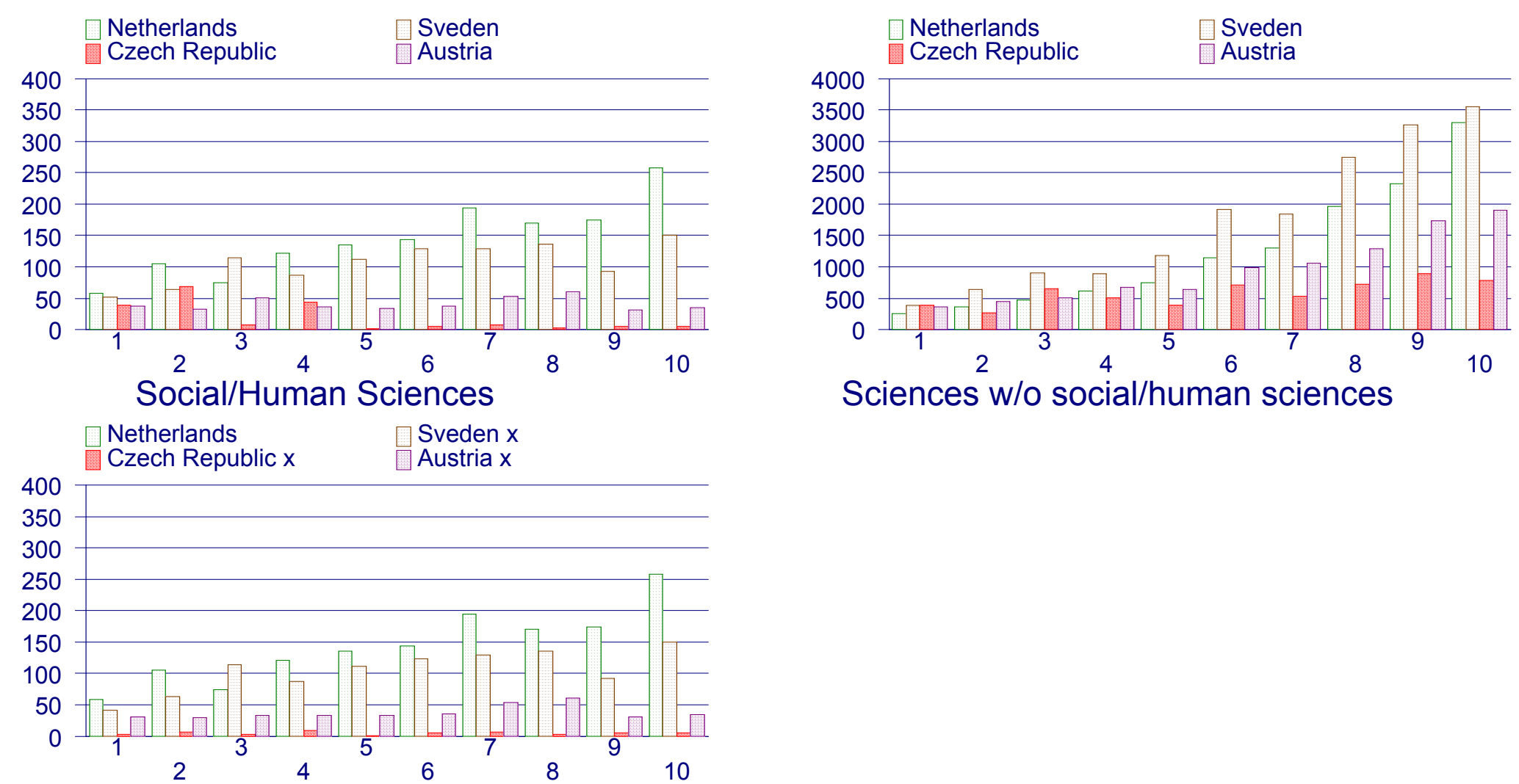

Social/Human Sciences [x-w/o national journals]

\section{Decile groups of journal's impact factors}


Table 2. Number of articles in IF journals produced by authors with an affiliation in the Czech Republic (social sciences and humanities)

\begin{tabular}{|c|c|c|c|}
\hline \multirow[b]{2}{*}{$\begin{array}{l}\text { Social sciences and humanities according to } \\
\text { ISI Thomson. }\end{array}$} & \multicolumn{2}{|c|}{ Journal } & \multirow[b]{2}{*}{ Total } \\
\hline & Foreign & Domestic & \\
\hline ANTHROPOLOGY & 4 & 0 & 4 \\
\hline BUSINESS, FINANCE & 1 & 12 & 13 \\
\hline ECONOMICS & 11 & 43 & 54 \\
\hline ENVIRONMENTAL STUDIES & 2 & 0 & 2 \\
\hline ETHICS & 1 & 0 & 1 \\
\hline INFORMATION SCIENCE & 2 & 0 & 2 \\
\hline INTERNATIONAL RELATIO & 4 & 0 & 4 \\
\hline LAW & 1 & 0 & 1 \\
\hline LINGUISTICS & 1 & 0 & 1 \\
\hline POLITICAL SCIENCE & 7 & 0 & 7 \\
\hline PSYCHIATRY & 7 & 0 & 7 \\
\hline PSYCHOLOGY, BIOLOGICAL & 2 & 0 & 2 \\
\hline PSYCHOLOGY, CLINICAL & 1 & 0 & 1 \\
\hline PSYCHOLOGY, DEVELOPMENTAL & 1 & 0 & 1 \\
\hline PSYCHOLOGY, MULTIDISC & 2 & 44 & 46 \\
\hline PSYCHOLOGY, SOCIAL & 1 & 0 & 1 \\
\hline PUBLIC ADMINISTRATION & 1 & 0 & 1 \\
\hline PUBLIC, ENVIRONMENTAL & 1 & 0 & 1 \\
\hline SOCIAL ISSUES & 1 & 0 & 1 \\
\hline SOCIAL SCIENCES, INTE & 2 & 0 & 2 \\
\hline SOCIOLOGY & 2 & 38 & 40 \\
\hline SUBSTANCE ABUSE & 2 & 0 & 2 \\
\hline Total & 57 & 137 & 194 \\
\hline
\end{tabular}


Figure 2. Number of articles in IF journals published in 2006 (weighted on population of the Czech Republic)

Number of articles in 2006 [country population size adjusted] ( $x$ - CR, AU, SW w/o national journals)
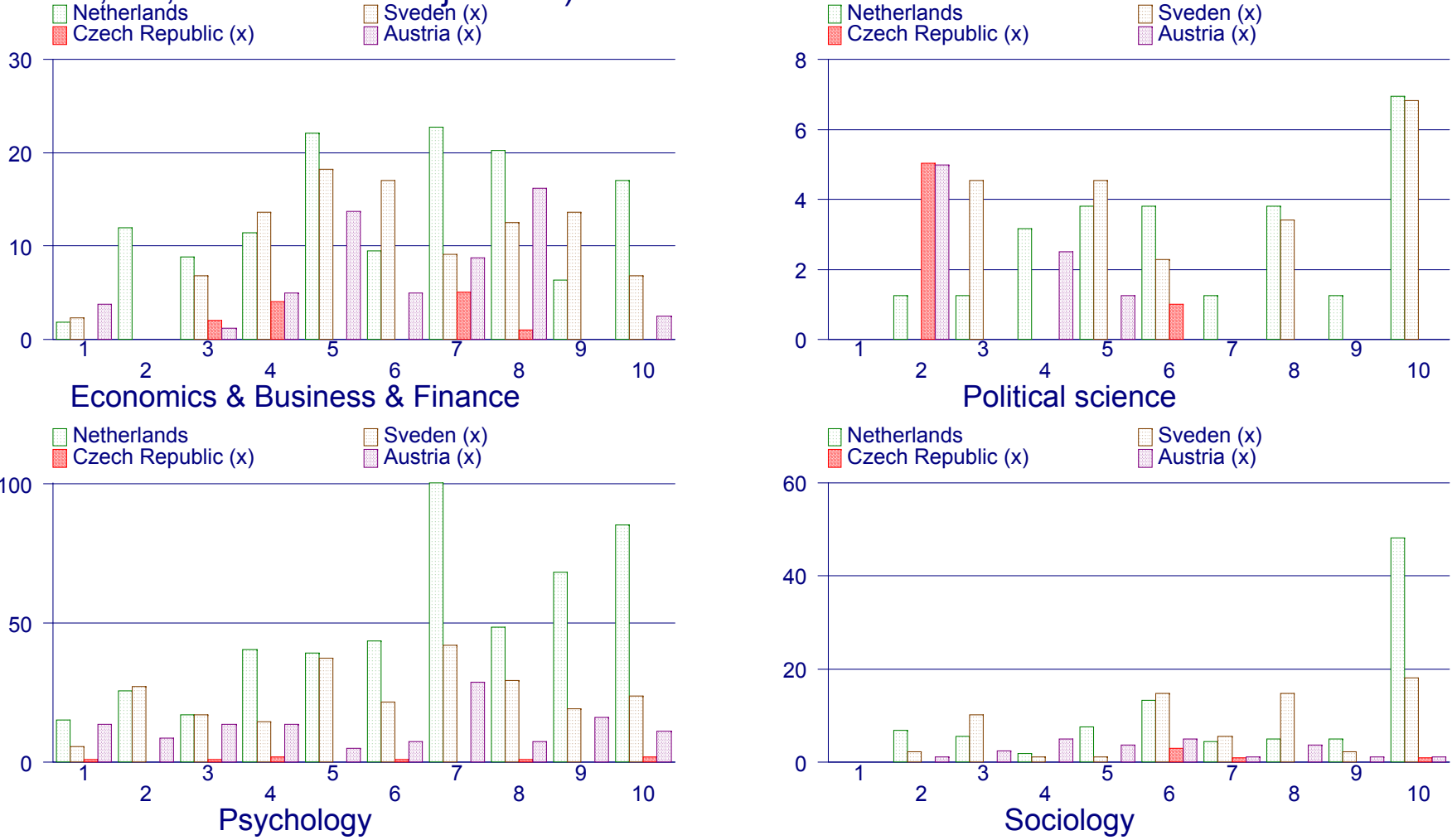

lower impact factor <--- 10 deciles ---> higher impact

Decile groups of journals by impact factor 
Table 3. Authors with highest value of total impact factor (IF sum) in foreign and all languages journals - narrower and broader group of sociology. Period 19982007.

\begin{tabular}{|c|c|c|c|c|c|c|c|c|c|}
\hline \multirow[t]{4}{*}{ Author } & \multirow[t]{4}{*}{ Affiliation } & \multicolumn{4}{|c|}{ IF sum } & \multicolumn{4}{|c|}{ Rank } \\
\hline & & \multicolumn{2}{|c|}{$\begin{array}{c}\text { Foreign } \\
\text { language }\end{array}$} & \multicolumn{2}{|c|}{$\begin{array}{c}\text { All } \\
\text { languages }\end{array}$} & & & & \\
\hline & & 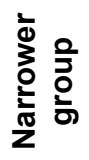 & $\begin{array}{l}\frac{1}{ \pm} \\
\frac{0}{0} \\
\frac{0}{0} \\
\frac{0}{0}\end{array}$ & 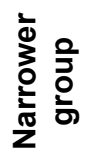 & 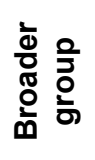 & & & & \\
\hline & & $(1)$ & (2) & (3) & (4) & $\left(1^{\prime}\right)$ & $\left(2^{\prime}\right)$ & (3) & $\left(4^{\prime}\right)$ \\
\hline Vecernik, J. & SOÚ AV ČR & 1.2 & 2.5 & 2.0 & 4.4 & 1 & 1 & 2 & 1 \\
\hline Kreidl, M. & FF ZČU & 1.2 & 1.2 & 2.0 & 2.0 & 2 & 4 & 1 & 3 \\
\hline Simonova, N. & SOÚ AV ČR & 0.8 & 0.8 & 1.0 & 1.0 & 3 & 8 & 10 & 16 \\
\hline Mateju, P. & SOÚ AV ČR & 0.7 & 1.0 & 1.2 & 1.5 & 4 & 7 & 5 & 7 \\
\hline Strakova, J. & SOÚ AV ČR & 0.6 & 0.6 & 0.8 & 0.8 & 5 & 11 & 14 & 21 \\
\hline Illner, M. & SOÚ AV ČR & 0.6 & 0.6 & 0.7 & 0.7 & 6 & 12 & 18 & 26 \\
\hline Hamplova, D. & SOÚ AV ČR & 0.5 & 0.5 & 0.9 & 0.9 & 7 & 13 & 12 & 18 \\
\hline Nespor, Z.R. & SOÚ AV ČR & 0.5 & 0.5 & 1.5 & 1.5 & 8 & 14 & 3 & 6 \\
\hline Kuchar, P. & FSV UK & 0.4 & 0.7 & 0.4 & 0.7 & 9 & 10 & 27 & 22 \\
\hline Fonadova, L. & ESF MU & 0.3 & 0.3 & 0.3 & 0.3 & 10 & 17 & 32 & 37 \\
\hline Katrnak, T. & FSS MU & 0.3 & 0.3 & 1.0 & 1.0 & 11 & 18 & 7 & 14 \\
\hline Krejci, J. & SOÚ AV ČR & 0.3 & 0.3 & 0.7 & 0.7 & 12 & 19 & 17 & 24 \\
\hline Kucerova, E. & ČZU & 0.3 & 0.4 & 0.3 & 0.4 & 13 & 15 & 35 & 30 \\
\hline Linek, L. & SOÚ AV ČR & 0.3 & 0.3 & 0.3 & 0.3 & 14 & 21 & 39 & 45 \\
\hline Rabusic, L. & FSS MU & 0.3 & 0.3 & 1.2 & 1.2 & 15 & 22 & 6 & 13 \\
\hline Keller, J. & ZSF OSU & 0.2 & 0.2 & 0.4 & 0.4 & 16 & 25 & 28 & 36 \\
\hline Benacek, V. & SOÚ AV ČR & 0.2 & 0.2 & 0.2 & 1.3 & 17 & 26 & 59 & 11 \\
\hline Dudova, R. & SOÚ AV ČR & 0.2 & 0.2 & 0.2 & 0.2 & 18 & 27 & 62 & 68 \\
\hline Haskova, $\mathrm{H}$. & SOÚ AV ČR & 0.2 & 0.2 & 0.3 & 0.3 & 19 & 28 & 33 & 38 \\
\hline Kolarova, M. & SOÚ AV ČR & 0.2 & 0.2 & 0.2 & 0.2 & 20 & 29 & 63 & 69 \\
\hline Lyons, $\mathrm{P}$. & SOÚ AV ČR & 0.2 & 0.3 & 0.2 & 0.3 & 21 & 20 & 65 & 42 \\
\hline Mansfeldova, Z. & SOÚ AV ČR & 0.2 & 0.2 & 0.2 & 0.2 & 22 & 30 & 44 & 50 \\
\hline Nesporova, O. & VÚPSV & 0.2 & 0.2 & 0.2 & 0.2 & 23 & 31 & 68 & 69 \\
\hline Sirovatka, T. & FSS MU & 0.2 & 1.0 & 0.6 & 2.2 & 24 & 6 & 19 & 2 \\
\hline Vail, B.J. & FSS MU & 0.2 & 0.2 & 0.2 & 0.2 & 25 & 32 & 53 & 62 \\
\hline Tucek, M. & SOÚ AV ČR & 0.1 & 0.1 & 0.1 & 0.1 & 26 & 34 & 78 & 72 \\
\hline Rakusanova, $\mathrm{P}$. & SOÚ AV ČR & 0.1 & 0.1 & 0.1 & 0.1 & 27 & 36 & 84 & 92 \\
\hline Rabusicova, M. & FF MU & 0.1 & 0.1 & 0.1 & 0.1 & 28 & 35 & 89 & 91 \\
\hline Konopasek, Z. & FSS MU & 0.1 & 0.1 & 0.1 & 0.1 & 29 & 39 & 72 & 77 \\
\hline Nekola, M. & FSV UK & 0.1 & 0.1 & 0.1 & 0.1 & 30 & 37 & 98 & 103 \\
\hline Rehakova, B. & SOÚ AV ČR & 0.1 & 0.1 & 1.4 & 1.4 & 31 & 38 & 4 & 10 \\
\hline Vesely, A. & FSV UK & & 1.4 & 0.2 & 1.5 & & 2 & 66 & 5 \\
\hline Lux, M. & SOÚ AV ČR & & 1.3 & 0.5 & 2.0 & & 3 & 23 & 4 \\
\hline Potucek, M. & FSV UK & & 1.2 & 0.1 & 1.2 & & 5 & 86 & 12 \\
\hline Vlachova, K. & SOÚ AV ČR & & 0.8 & 0.6 & 1.4 & & 9 & 21 & 9 \\
\hline Mares, P. & FSS MU & & 0.4 & 1.0 & 1.5 & & 16 & 8 & 8 \\
\hline Musil, J. & FSV UK & & & 1.0 & 1.0 & & & 9 & 15 \\
\hline Kostelecky, T. & SOÚ AV ČR & & & 0.9 & 0.9 & & & 11 & 17 \\
\hline
\end{tabular}

Note. This table presents 31 authors with highest value of total impact factor (according to foreign language category, narrower group - IF sum value 0.05 and higher before rounding). In addition, authors who represent "first ten" in one of the other categories are also included.

Rank of authors is based on IF sum for a given category. For authors with the same IF sum value, number of articles taken into account for analysis was used and also alphabetical order if needed. 
Table 4. Forty year old authors and younger (born 1968 and later) with highest value of total impact factor (IF sum) according to journals in foreign language and in all languages - narrower and broader group of sociology journals.

\begin{tabular}{|c|c|c|c|c|c|c|c|c|c|}
\hline \multirow[t]{4}{*}{ Author } & \multirow[t]{4}{*}{ Affiliation } & \multicolumn{4}{|c|}{ IF sum } & \multicolumn{4}{|c|}{ Rank } \\
\hline & & \multicolumn{2}{|c|}{$\begin{array}{c}\text { Foreign } \\
\text { language }\end{array}$} & \multicolumn{2}{|c|}{$\begin{array}{c}\text { All } \\
\text { languages }\end{array}$} & & & & \\
\hline & & 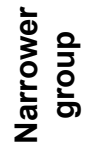 & 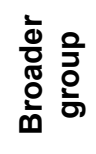 & 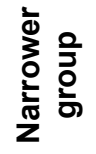 & $\begin{array}{ll}\frac{1}{0} & 0 \\
\frac{\pi}{7} & 0 \\
0 & 0 \\
\frac{1}{0} & 0\end{array}$ & & & & \\
\hline & & (1) & (2) & (3) & (4) & (1') & $\left(2^{\prime}\right)$ & $\left(3^{\prime}\right)$ & $\left(4^{\prime}\right)$ \\
\hline Kreidl, M. & FF ZČU & 1.2 & 1.2 & 2.0 & 2.0 & 1 & 3 & 1 & 1 \\
\hline Simonova, N. & SOÚ AV ČR & 0.8 & 0.8 & 1.0 & 1.0 & 2 & 4 & 4 & 7 \\
\hline Hamplova, D. & SOÚ AV ČR & 0.5 & 0.5 & 0.9 & 0.9 & 3 & 6 & 5 & 8 \\
\hline Nespor, Z.R. & SOÚ AV ČR & 0.5 & 0.5 & 1.5 & 1.5 & 4 & 7 & 2 & 4 \\
\hline Katrnak, T. & FSS MU & 0.3 & 0.3 & 1.0 & 1.0 & 5 & 9 & 3 & 6 \\
\hline Krejci, J. & SOÚ AV ČR & 0.3 & 0.3 & 0.7 & 0.7 & 6 & 10 & 6 & 10 \\
\hline Kucerova, E. & ČZU & 0.3 & 0.4 & 0.3 & 0.4 & 7 & 8 & 15 & 13 \\
\hline Linek, L. & SOÚ AV ČR & 0.3 & 0.3 & 0.3 & 0.3 & 8 & 12 & 17 & 19 \\
\hline Dudova, R. & SOÚ AV ČR & 0.2 & 0.2 & 0.2 & 0.2 & 9 & 14 & 22 & 24 \\
\hline Haskova, H. & SOÚ AV ČR & 0.2 & 0.2 & 0.3 & 0.3 & 10 & 15 & 13 & 15 \\
\hline Kolarova, M. & SOÚ AV ČR & 0.2 & 0.2 & 0.2 & 0.2 & 11 & 16 & 25 & 25 \\
\hline Lyons, $\mathrm{P}$. & SOÚ AV ČR & 0.2 & 0.3 & 0.2 & 0.3 & 12 & 11 & 27 & 17 \\
\hline Nesporova, O. & VÚPSV & 0.2 & 0.2 & 0.2 & 0.2 & 13 & 17 & 28 & 27 \\
\hline Vail, B.J. & FSS MU & 0.2 & 0.2 & 0.2 & 0.2 & 14 & 18 & 31 & 29 \\
\hline Rakusanova, $\mathrm{P}$. & SOÚ AV ČR & 0.1 & 0.1 & 0.1 & 0.1 & 15 & 19 & 38 & 38 \\
\hline Nekola, M. & FSV UK & 0.1 & 0.1 & 0.1 & 0.1 & 16 & 20 & 43 & 43 \\
\hline Vesely, A. & FSV UK & & 1.4 & 0.2 & 1.5 & & 1 & 32 & 3 \\
\hline Lux, M. & SOÚ AV ČR & & 1.3 & 0.5 & 2.0 & & 2 & 10 & 2 \\
\hline Vlachova, $\mathrm{K}$. & SOÚ AV ČR & & 0.8 & 0.6 & 1.4 & & 5 & 8 & 5 \\
\hline Lebeda, T. & SOÚ AV ČR & & 0.2 & 0.6 & 0.8 & & 13 & 7 & 9 \\
\hline Rysavy, D. & FF UPOL & & & 0.5 & 0.5 & & & 9 & 12 \\
\hline Chaloupkova, J. & SOÚ AV ČR & & & 0.4 & 0.4 & & & 11 & 14 \\
\hline Sunega, $\mathrm{P}$. & SOÚ AV ČR & & & 0.3 & 0.5 & & & 14 & 11 \\
\hline
\end{tabular}

Note. This table presents 16 authors with the highest value of total impact factor (according to foreign language category, narrower group - IF sum value 0.05 and higher before rounding). In addition, authors who represent "top ten" in one of the other categories are also included.

Rank of authors is based on IF sum for a given category. For authors with the same IF sum value, number of articles taken into account for analysis was used and also alphabetical order if needed. 
Table 5. Authors with highest citation characteristics according to Web of Science within the 1998-2007 period and without period limitations

\begin{tabular}{|c|c|c|c|c|c|c|c|c|c|c|c|c|}
\hline \multirow[b]{2}{*}{ 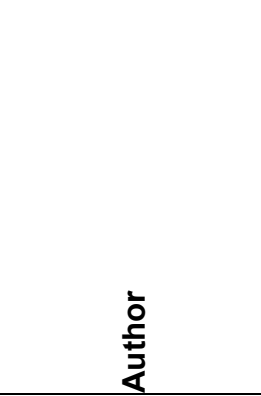 } & \multirow[b]{2}{*}{ 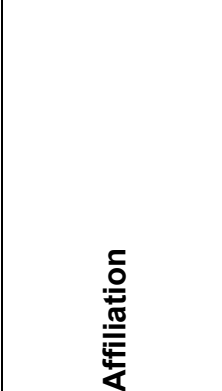 } & \multicolumn{5}{|c|}{$\begin{array}{l}\text { (A) Data on articles from } \\
1998-2007 \text { period }\end{array}$} & \multicolumn{5}{|c|}{$\begin{array}{l}\text { (B) Data on articles without } \\
\text { period limitations }\end{array}$} & \multirow{3}{*}{ 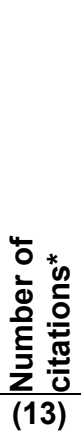 } \\
\hline & & 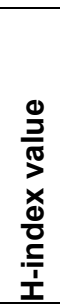 & 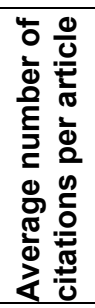 & 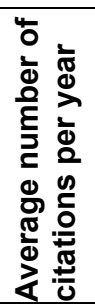 & 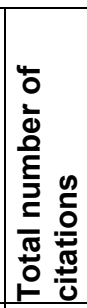 & 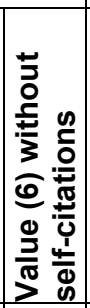 & $\begin{array}{l}\stackrel{0}{J} \\
\frac{0}{\pi} \\
\frac{x}{0} \\
\frac{0}{0} \\
\cdot \frac{1}{1} \\
\text { I }\end{array}$ & 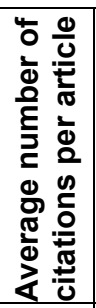 & 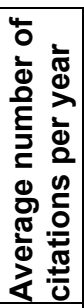 & 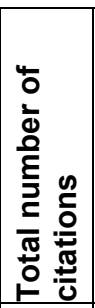 & 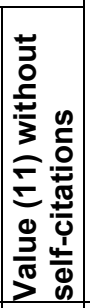 & \\
\hline & (2) & (3) & $(4)$ & (5) & $(6)$ & $(7)$ & $(8)$ & (9) & (10) & (11) & \begin{tabular}{|l|}
$(12)$ \\
\end{tabular} & \\
\hline Mateju, P. & SOÚ AV ČR & 3 & 2.46 & 2.91 & 32 & 23 & 6 & 2.91 & 3.54 & 99 & 65 & 70 \\
\hline Vlachova, K. & SOÚ AV ČR & 3 & 2.62 & 1.91 & 21 & 11 & 4 & 1.69 & 1.80 & 27 & 16 & 32 \\
\hline Kostelecky, T. & SOÚ AV ČR & 3 & 2.12 & 1.55 & 17 & 9 & & & & & & 53 \\
\hline Rehak, J. & SC\&C & 3 & 4.67 & 1.27 & 14 & 4 & & & & & & 12 \\
\hline Vecernik, J. & SOÚ AV ČR & 2 & 1.48 & 2.82 & 31 & 22 & 4 & 1.26 & 2.04 & 53 & 41 & 160 \\
\hline Rabusic, L. & FSS MU & 2 & 1.60 & 1.45 & 16 & 15 & 3 & 2.11 & 2.11 & 38 & 32 & 49 \\
\hline Kreidl, M. & FF ZČU & 2 & 1.30 & 1.30 & 13 & 8 & & & & & & 40 \\
\hline Sirovatka, T. & FSS MU & 2 & 0.92 & 1.09 & 12 & 8 & & & & & & 33 \\
\hline Cermak, D. & SOÚ AV ČR & 2 & 4.00 & 1.60 & 8 & 7 & & & & & & 7 \\
\hline Rehakova, B. & SOÚ AV ČR & 2 & 0.64 & 0.64 & 7 & 6 & 4 & 1.80 & 1.55 & 45 & 34 & 16 \\
\hline Vajdova, Z. & SOÚ AV ČR & 2 & 2.33 & 0.64 & 7 & 6 & 3 & 2.50 & 0.94 & 15 & 9 & 9 \\
\hline Mares, P. & FSS MU & 2 & 0.67 & 0.55 & 6 & 6 & 3 & 1.50 & 0.92 & 24 & 19 & 56 \\
\hline Simonova, N. & SOÚ AV ČR & 2 & 1.00 & 0.83 & 5 & 5 & & & & & & 4 \\
\hline Kuchar, P. & FSV UK & 2 & 2.00 & 0.67 & 6 & 5 & & & & & & 7 \\
\hline Burianek, J. & FF UK & 2 & 1.20 & 0.55 & 6 & 5 & & & & & & 12 \\
\hline Hamplova, D. & SOÚ AV ČR & 2 & 1.00 & 0.62 & 5 & 4 & & & & & & 22 \\
\hline Nespor, Z.R. & SOÚ AV ČR & 2 & 1.00 & 1.67 & 10 & 4 & & & & & & 25 \\
\hline Lux, M. & SOÚ AV ČR & 2 & 0.86 & 0.67 & 6 & 2 & & & & & & 53 \\
\hline Potucek, M. & FSV UK & 1 & 1.75 & 0.64 & 7 & 7 & 2 & 1.38 & 0.62 & 18 & 15 & 36 \\
\hline Strakova, J. & SOÚ AV ČR & 1 & 1.20 & 1.20 & 6 & 5 & & & & & & 8 \\
\hline Kucerova, E. & ČZU & 1 & 2.50 & 1.67 & 5 & 5 & & & & & & 1 \\
\hline Musil, J. & FSV UK & 1 & 1.00 & 0.50 & 5 & 5 & 4 & 3.56 & 2.04 & 57 & 54 & 85 \\
\hline Soukup, $P$. & FSV UK & 1 & 1.00 & 1.00 & 2 & 2 & & & & & & 2 \\
\hline Illner, M. & SOÚ AV ČR & 1 & 3.00 & 0.27 & 3 & 3 & 3 & 1.30 & 0.52 & 13 & 11 & 29 \\
\hline Katrnak, T. & FSS MU & 1 & 0.71 & 0.45 & 5 & 3 & & & & & & 18 \\
\hline Benacek, V. & SOÚ AV ČR & 1 & 0.60 & 0.33 & 3 & 3 & 3 & 1.17 & 0.52 & 14 & 11 & 32 \\
\hline Hamanova, J. & SC\&C & 1 & 3.00 & 0.27 & 3 & 3 & & & & & & 5 \\
\hline Bartova, I. & SC\&C & 1 & 3.00 & 0.27 & 3 & 3 & & & & & & 2 \\
\hline Hermanova, E. & n.a.(SOU AV) & 1 & 3.00 & 0.33 & 3 & 3 & 2 & 3.50 & 0.44 & 7 & 7 & 4 \\
\hline Vyhlidal, J. & VÚPSV & 1 & 3.00 & 0.60 & 3 & 3 & & & & & & 3 \\
\hline Chaloupkova, J. & SOÚ AV ČR & 1 & 1.00 & 0.75 & 3 & 3 & & & & & & 3 \\
\hline Haskova, $\mathrm{H}$. & SOÚ AV ČR & 1 & 1.00 & 0.25 & 2 & 2 & & & & & & 17 \\
\hline Tucek, M. & SOÚ AV ČR & 1 & 2.00 & 0.67 & 2 & 2 & 2 & 1.57 & 0.96 & 22 & 15 & 20 \\
\hline Nekola, M. & FSV UK & 1 & 2.00 & 0.67 & 2 & 2 & & & & & & 2 \\
\hline Zizlavsky, M. & n.a.(FSS MU) & 1 & 1.00 & 0.29 & 2 & 2 & & & & & & 2 \\
\hline Brokl, L. & FF UHK & 1 & 0.33 & 0.16 & 2 & 2 & 2 & 1.33 & 0.42 & 8 & 7 & 11 \\
\hline Louckova, I. & FF UPOL & 1 & 2.00 & 0.29 & 2 & 2 & 2 & 1.75 & 0.27 & 7 & 5 & 5 \\
\hline Kabele, J. & FSV UK & 1 & 2.00 & 0.67 & 2 & 2 & 3 & 2.70 & 1.12 & 27 & 22 & 10 \\
\hline Lebeda, $\mathrm{T}$. & SOÚ AV ČR & 1 & 1.00 & 0.71 & 5 & 2 & & & & & & 24 \\
\hline Vesely, A. & FSV UK & 1 & 1.00 & 0.40 & 2 & 2 & & & & & & 5 \\
\hline
\end{tabular}




\begin{tabular}{|l|l|l|l|l|l|l|l|l|l|l|c|c|}
\hline Linek, L. & SOÚ AV ČR & 1 & 1.00 & 0.50 & 2 & 1 & & & & & & 23 \\
\hline Krejci, J. & SOÚ AV ČR & 1 & 1.50 & 0.50 & 3 & 1 & 2 & 1.50 & 0.86 & 6 & 1 & 19 \\
\hline Mansfeldova, Z. & SOÚ AV ČR & 1 & 1.00 & 0.29 & 2 & 1 & 2 & 0.83 & 0.19 & 5 & 3 & 29 \\
\hline Lyons, P. & SOÚ AV ČR & 1 & 0.50 & 0.33 & 1 & 1 & & & & & & 3 \\
\hline Rakusanova, P. & SOÚ AV ČR & 1 & 2.00 & 0.50 & 2 & 1 & & & & & & 8 \\
\hline Konopasek, Z. & FSS MU & 1 & 0.67 & 0.20 & 2 & 1 & 2 & 0.57 & 0.22 & 4 & 3 & 14 \\
\hline Louzek, M. & FF UK & 1 & 0.07 & 0.10 & 1 & 1 & & & & & & 31 \\
\hline Novak, M. & FSV UK & 1 & 0.40 & 0.18 & 2 & 1 & & & & & & 36 \\
\hline Kepakova, K. & n.a.(FSS MU) & 1 & 1.00 & 0.10 & 1 & 1 & & & & & & 1 \\
\hline Vojtiskova, K. & SOÚ AV ČR & 1 & 2.00 & 0.67 & 2 & 1 & & & & & & 0 \\
\hline Sunega, P. & SOÚ AV ČR & 1 & 0.75 & 0.50 & 3 & 1 & & & & & & 15 \\
\hline Stachova, J. & SOÚ AV ČR & 1 & 1.00 & 0.25 & 1 & 1 & & & & & & 4 \\
\hline Fialova, L. & FTVS UK & 1 & 1.00 & 0.20 & 1 & 1 & & & & & & 9 \\
\hline Hudeckova, H. & PEF ČZU & 1 & 1.00 & 0.14 & 1 & 1 & & & & & & 24 \\
\hline Lostak, M. & PEF ČZU & 1 & 1.00 & 0.14 & 1 & 1 & & & & & & 7 \\
\hline Slepickova, L. & FSS MU & 1 & 1.00 & 0.50 & 1 & 1 & & & & & & 0 \\
\hline Pridalova, M. & FSS MU & 1 & 1.00 & 0.09 & 1 & 1 & & & & & & 3 \\
\hline Nedbalkova, K. & FSS MU & 1 & 0.50 & 0.11 & 1 & 1 & & & & & & 2 \\
\hline Marada, R. & FSS MU & 1 & 1.00 & 0.12 & 1 & 1 & & & & & & 8 \\
\hline Sanderova, J. & FSV UK & 1 & 1.00 & 0.10 & 1 & 1 & & & & & & 6 \\
\hline Petrusek, M. & FSV UK & 1 & 0.25 & 0.14 & 1 & 1 & 2 & 0.71 & 0.48 & 10 & 10 & 12 \\
\hline Fonadova, L. & ESF MU & 1 & 0.50 & 0.20 & 1 & 0 & & & & & & 1 \\
\hline Jerabek, H. & FSV UK & 1 & 0.50 & 0.22 & 2 & 0 & 2 & 0.62 & 0.30 & 8 & 2 & 9 \\
\hline Keller, J. & ZSF OSU & 0 & 0.00 & 0.00 & 0 & 0 & 1 & 0.56 & 0.23 & 5 & 3 & 49 \\
\hline Fric, P. & FSV UK & 0 & 0.00 & 0.00 & 0 & 0 & 1 & 0.50 & 0.14 & 3 & 3 & 37 \\
\hline Cermakova, M. & SOÚ AV ČR & 0 & 0.00 & 0.00 & 0 & 0 & 2 & 2.00 & 0.42 & 8 & 6 & 24 \\
\hline Havelka, M. & FHS UK & 0 & 0.00 & 0.00 & 0 & 0 & 1 & 0.38 & 0.14 & 3 & 3 & 12 \\
\hline Mozny, I. & FSS MU & 0 & 0.00 & 0.00 & 0 & 0 & 1 & 0.25 & 0.07 & 1 & 1 & 27 \\
\hline
\end{tabular}

Notes:

This table includes authors with h-index value 1 and higher.

Data do not reflect co-authorship.

Search was carried out using limitations on "Articles" (document type).

Authors are presented in descending order according to H-index in column (3) and next according to column (7). Expression "n.a." in affiliation column means that current affiliation is not available and the one mentioned within articles found is in brackets.

In part (B) "without period limitations" only data for authors with higher h-index value (compared to 1998-2007 period) are presented. In addition, the table includes authors with "0" h-index value in 1998-2007 period but with h-index value of 1 or 2 for part (B) and with value 10 and higher in column (13).

* For 1998-2006 period as there were not complete data for 2007 when this paper was written. It is based on information available in Web of Knowledge using Cited Reference Search (represents citations of all document types - articles, monographs etc.). 
Table 6. Characteristics of the most frequently cited articles (from those taken into account for our analyses)

\begin{tabular}{|c|c|c|c|c|c|c|c|c|c|}
\hline & & & Nur & ber of cit & ations & & & & \\
\hline & Author(s) & & & & & & 등 & ह을 & \\
\hline Hraba, J. & McCutcheon, A. & Vecernik, J. & 11 & 2 & 2 & RURAL SOCIOL & 64 & 3 & 1999 \\
\hline Vlachova, K. & Mateju, P. & & 6 & 3 & 0 & SOCIOL CAS & 34 & 2 & 1998 \\
\hline Rabusic, L. & & & 5 & 5 & 1 & SOCIOL CAS & 36 & 1 & 2000 \\
\hline Kreidl, M. & Vlachova, K. & & 5 & 2 & 1 & SOCIOL CAS & 35 & 3 & 1999 \\
\hline Kostelecky, T. & Cermak, D. & & 5 & 5 & 1 & SOCIOL CAS & 40 & 4 & 2004 \\
\hline Mateju, P. & Vlachova, K. & & 5 & 3 & 0 & SOCIOL CAS & 34 & 2 & 1998 \\
\hline Vecernik, J. & & & 5 & 3 & 3 & $\begin{array}{l}\text { COMMUNIS POST- } \\
\text { COMMUN }\end{array}$ & 32 & 4 & 1999 \\
\hline Potucek, M. & & & 5 & 5 & 4 & J EUR SOC POLICY & 14 & 3 & 2004 \\
\hline Rehak, J. & & & 5 & 5 & 0 & SOCIOL CAS & 34 & 1 & 1998 \\
\hline
\end{tabular}

Note: Data from the Web of Science were collected by 31.5. 2008. 


\section{Appendix}

Table A1. List of journals and their IF - data for 2007 according to the WoS $^{* 1}$ )

\begin{tabular}{|c|c|c|c|c|}
\hline $\begin{array}{c}\text { Journal title } \\
\text { (abbreviated) }\end{array}$ & Journal title (full) & $\begin{array}{c}\text { IF } \\
\text { value }\end{array}$ & \begin{tabular}{|c|} 
Subject \\
category (see \\
table A2)
\end{tabular} & $\begin{array}{l}\text { Narrower } \\
\text { group }^{* 2)}\end{array}$ \\
\hline SOCIOL CAS & $\begin{array}{l}\text { SOCIOLOGICKY CASOPIS/CZECH } \\
\text { SOCIOLOGICAL REVIEW }\end{array}$ & 0.169 & 1 & Yes \\
\hline SOCIOLOGIA & SOCIOLOGIA & 0.116 & 1 & Yes \\
\hline SOTSIOL ISSLED & SOTSIOLOGICHESKIE ISSLEDOVANIYA & 0.194 & 1 & Yes \\
\hline EUR SOCIOL REV & EUROPEAN SOCIOLOGICAL REVIEW & 0.855 & 1 & Yes \\
\hline SOCIOL RURALIS & SOCIOLOGIA RURALIS & 0.641 & 1 & Yes \\
\hline INT J COMP SOCIOL & $\begin{array}{l}\text { INTERNATIONAL JOURNAL OF } \\
\text { COMPARATIVE SOCIOLOGY }\end{array}$ & $0.121^{3+1}$ & 1 & Yes \\
\hline POL SOCIOL REV & POLISH SOCIOLOGICAL REVIEW & 0.021 & 1 & Yes \\
\hline SOCIOL QUART & SOCIOLOGICAL QUARTERLY & 0.627 & 1 & Yes \\
\hline RURAL SOCIOL & RURAL SOCIOLOGY & 0.820 & 1 & Yes \\
\hline BRIT J SOCIOL EDUC & $\begin{array}{l}\text { BRITISH JOURNAL OF SOCIOLOGY OF } \\
\text { EDUCATION }\end{array}$ & 0.609 & 4,1 & Yes \\
\hline SOCIOL EDUC & SOCIOLOGY OF EDUCATION & 1.290 & 4,1 & Yes \\
\hline SOC INDIC RES & SOCIAL INDICATORS RESEARCH & 0.610 & $2,9,1$ & Yes \\
\hline WORK EMPLOY SOC & WORK EMPLOYMENT AND SOCIETY & 1.051 & $6,15,1$ & Yes \\
\hline J POLIT MIL SOCIOL & $\begin{array}{l}\text { JOURNAL OF POLITICAL \& MILITARY } \\
\text { SOCIOLOGY }\end{array}$ & 0.061 & 7,1 & Yes \\
\hline HUM STUD & HUMAN STUDIES & 0.128 & 12,1 & Yes \\
\hline FINANC UVER & FINANCE A UVER & 0.382 & 5 & No \\
\hline POLIT EKON & POLITICKA EKONOMIE & 0.172 & 6,7 & No \\
\hline FILOS CAS & FILOSOFICKY CASOPIS & 0.011 & 12,13 & No \\
\hline EUR J POLIT RES & $\begin{array}{l}\text { EUROPEAN JOURNAL OF POLITICAL } \\
\text { RESEARCH }\end{array}$ & 1.679 & 7 & No \\
\hline CESK PSYCHOL & CESKOSLOVENSKA PSYCHOLOGIE & 0.133 & 10,8 & No \\
\hline JUSTICE SYST J & JUSTICE SYSTEM JOURNAL & 0.122 & 17 & No \\
\hline JEUR SOC POLICY & $\begin{array}{l}\text { JOURNAL OF EUROPEAN SOCIAL } \\
\text { POLICY }\end{array}$ & 1.083 & 3 & No \\
\hline OSTEUROPA & OSTEUROPA & 0.223 & 7 & No \\
\hline POLIT STUD-LONDON & POLITICAL STUDIES & 0.488 & 7 & No \\
\hline SOC POLICY ADMIN & SOCIAL POLICY \& ADMINISTRATION & 0.512 & 16,3 & No \\
\hline $\begin{array}{l}\text { COMMUNIS POST- } \\
\text { COMMUN }\end{array}$ & $\begin{array}{l}\text { COMMUNIST AND POST-COMMUNIST } \\
\text { STUDIES }\end{array}$ & 0.529 & 11,7 & No \\
\hline REV ETUD COMP EST-O & $\begin{array}{l}\text { REVUE D ETUDES COMPARATIVES } \\
\text { ESTOUEST }\end{array}$ & 0.071 & 6 & No \\
\hline EASTERN EUR ECON & EASTERN EUROPEAN ECONOMICS & 0.413 & 6 & No \\
\hline $\begin{array}{l}\text { COMMUNIS POST- } \\
\text { COMMUN }\end{array}$ & $\begin{array}{l}\text { COMMUNIST AND POST-COMMUNIST } \\
\text { STUDIES }\end{array}$ & 0.529 & 11,7 & No \\
\hline BRIT J PSYCHIAT & BRITISH JOURNAL OF PSYCHIATRY & 5.446 & 18 & No \\
\hline PERCEPT MOTOR SKILL & PERCEPTUAL AND MOTOR SKILLS & 0.387 & 10 & No \\
\hline URBAN STUD & URBAN STUDIES & 1.274 & 19 & No \\
\hline PHIL TODAY & PHILOSOPHY TODAY & & 13 & No \\
\hline HIST JAHR & HISTORISCHES JAHRBUCH & 4) & 14 & No \\
\hline HIST SOC RES & $\begin{array}{l}\text { HISTORICAL SOCIAL RESEARCH- } \\
\text { HISTORISCHE SOZIALFORSCHUNG }\end{array}$ & (4) & 14,15 & No \\
\hline SWS-RUNDSCHAU & SWS-RUNDSCHAU & 4) & 7 & No \\
\hline ESPRIT & ESPRIT & & 20 & No \\
\hline
\end{tabular}

*1) Only journals that appeared at least once are listed.

*2) Narrower group represents journals which subject category includes category 1 .

*3) Data for 2002. Newer data are not available in the Web of Knowledge.

*4) Data was not found for 2007 or any previous year.

Note: For year 2007, within subject category "sociology", median IF $=0.631$; average IF $=$ 0.794 . 
Table A2. List of subject categories of journals in the Web of Knowledge*

\begin{tabular}{|c|l|}
\hline Code & \multicolumn{1}{|c|}{ Subject category } \\
\hline 1 & Sociology \\
\hline 2 & Social Sciences \\
\hline 3 & Social Issues \\
\hline 4 & Education \& Educational Research \\
\hline 5 & Business, Finance \\
\hline 6 & Economics \\
\hline 7 & Political Science \\
\hline 8 & Multidisciplinary \\
\hline 9 & Interdisciplinary \\
\hline 10 & Psychology \\
\hline 11 & International Relations \\
\hline 12 & Ethics \\
\hline 13 & Philosophy \\
\hline 14 & History \\
\hline 15 & Industrial Relations \& Labor \\
\hline 16 & Planning \& Development \\
\hline 17 & Law \\
\hline 18 & Psychiatry \\
\hline 19 & Environmental Studies, Urban Studies \\
\hline 20 & Other \\
\hline
\end{tabular}

* Only categories that appeared at least once are listed. 
Table A3. List of institutes/departments addressed for cooperation

\begin{tabular}{|c|c|}
\hline Abbreviation & Name \\
\hline ISS FSV UK * & $\begin{array}{l}\text { Institut sociologických studií - Fakulta sociálních věd UK [Institute of Sociological Studies, } \\
\text { Faculty of Social Sciences, Charles University] }\end{array}$ \\
\hline KS FF UK * & $\begin{array}{l}\text { Katedra sociologie - Filozofická fakulta UK [Deparment of Sociology, Faculty of } \\
\text { Philosophy and Arts, Charles University] }\end{array}$ \\
\hline FSS MU * & Fakulta sociálních studií MU [Faculty of Social Studies, Masaryk University] \\
\hline KSA FF UPOL * & $\begin{array}{l}\text { Katedra sociologie a andragogiky - Filozofická fakulta UPOL [Deparment of Sociology } \\
\text { and Education of the Edults, Philosophical Faculty, Palacký University Olomouc] }\end{array}$ \\
\hline KS FF ZČU * & $\begin{array}{l}\text { Katedra sociologie - Filozofická fakulta ZČU v Plzni [Deparment of Sociology, Faculty of } \\
\text { Philosophy and Arts, University of West Bohemia] }\end{array}$ \\
\hline KSP FSE UJEP & $\begin{array}{l}\text { Katedra sociální práce - Fakulta sociálně ekonomická, UJEP v Ústí nad Labem } \\
\text { [Department of Social Work, Faculty of Social and Economic Studies, Jan Evangelista } \\
\text { Purkyně University in Ústí nad Labem] }\end{array}$ \\
\hline SOÚ AV ČR * & $\begin{array}{l}\text { Sociologický ústav AV ČR, v.v.i. [Institute of Sociology, Academy of Sciences of the } \\
\text { Czech Republic] }\end{array}$ \\
\hline CESES FSV UK * & $\begin{array}{l}\text { Centrum pro sociální a ekonomické strategie - Fakulta sociálních věd UK [Center for } \\
\text { Social and Economic Strategies, Faculty of Social Sciences, Charles University] }\end{array}$ \\
\hline KSV FF UP * & $\begin{array}{l}\text { Katedra sociálních věd - Fakulta filozofická, Univerzita Pardubice [Department of Social } \\
\text { Sciences, Faculty of Arts and Philosophy, University of Pardubice] }\end{array}$ \\
\hline KREE EF VŠB-TU & $\begin{array}{l}\text { Katedra regionální a enviromentální ekonomiky - Ekonomická fakulta, Vysoká škola } \\
\text { báňská - Technická univerzita Ostrava [Department of Regional and Environmental } \\
\text { Economics, Faculty of Economics, VŠB - Technical University of Ostrava] }\end{array}$ \\
\hline KGS FHS UK & $\begin{array}{l}\text { Katedra genderových studií - Fakulta humanitních studií UK [Department of Gender } \\
\text { Studies, Faculty of Humanity Studies, Charles University] }\end{array}$ \\
\hline KSP ZSF OSU * & $\begin{array}{l}\text { Katedra sociální práce - Fakulta sociálních studií, Ostravská univerzita v Ostravě } \\
\text { [Department of Social Work, Faculty of Social Studies, University of Ostrava] }\end{array}$ \\
\hline KHV PEF ČZU * & $\begin{array}{l}\text { Katedra humanitních věd - Provozně ekonomická fakulta, Česká zemědělská univerzita } \\
\text { [Department of Humanities, Faculty of Economics and Management, Czech University of } \\
\text { Life Sciences Prague] }\end{array}$ \\
\hline ÚFSV FF UHK & $\begin{array}{l}\text { Ústav filosofie a společenských věd - Filozofická fakulta, Univerzita Hradec Králové } \\
\text { [Institute of Philosophy and Social Sciences, Faculty of Arts, University of Hradec } \\
\text { Králové] }\end{array}$ \\
\hline VÚPSV & $\begin{array}{l}\text { Výzkumný ústav práce a sociálních věcí, v.v.i. [Research Institute for Labour and Social } \\
\text { Affairs] }\end{array}$ \\
\hline KZKHV FTVS UK * & $\begin{array}{l}\text { Oddělení filosofie, historie a sociologie - Katedra základů kinantropologie a humanitních } \\
\text { věd - Fakulta tělesné výchovy a sportu UK [Department of Kinanthropology and } \\
\text { Humanities, Faculty of Physical Education and Sport, Charles University] }\end{array}$ \\
\hline KPSR̆ FPH VŠE & $\begin{array}{l}\text { Katedra psychologie a sociologie řízení - Fakulta podnikohospodářská, Vysoká škola } \\
\text { ekonomická [Department of psychology and sociology, University of Economics, Prague] }\end{array}$ \\
\hline
\end{tabular}

* We got a response from these. 
Table A4. List of the most frequently cited articles (from those taken into account for our analyses)

\begin{tabular}{|c|c|c|c|c|c|}
\hline Author(s) & Journal & Title of article & $\stackrel{\stackrel{0}{\xi}}{\frac{5}{0}}$ & 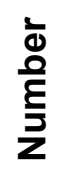 & Year \\
\hline $\begin{array}{l}\text { Hraba, J. } \\
\text { McCutcheon, } \\
\text { A. } \\
\text { Vecernik, J. }\end{array}$ & RURAL SOCIOL & $\begin{array}{l}\text { Rural and urban differences in economic } \\
\text { experiences, anxiety and support for the } \\
\text { post-communist reforms in the Czech and } \\
\text { Slovak Republics }\end{array}$ & 64 & 3 & 1999 \\
\hline $\begin{array}{l}\text { Vlachova, K. } \\
\text { Mateju, P. }\end{array}$ & SOCIOL CAS & $\begin{array}{l}\text { The crystallisation of political attitudes } \\
\text { and the political spectrum in the Czech } \\
\text { Republic }\end{array}$ & 34 & 2 & 1998 \\
\hline Rabusic, L. & SOCIOL CAS & Is Czech society 'post-materialist'? & 36 & 1 & 2000 \\
\hline $\begin{array}{l}\text { Kreidl, M. } \\
\text { Vlachova, K. }\end{array}$ & SOCIOL CAS & $\begin{array}{l}\text { The social background of the extreme } \\
\text { right in the Czech Republic }\end{array}$ & 35 & 3 & 1999 \\
\hline $\begin{array}{l}\text { Kostelecky, T. } \\
\text { Cermak, D. }\end{array}$ & SOCIOL CAS & $\begin{array}{l}\text { The influence of territorially specific } \\
\text { factors on the formation of the political } \\
\text { attitudes of voters }\end{array}$ & 40 & 4 & 2004 \\
\hline $\begin{array}{l}\text { Mateju, P } \\
\text { Vlachova, K. }\end{array}$ & SOCIOL CAS & $\begin{array}{l}\text { The role of politically relevant attitudes } \\
\text { and value orientations in electoral } \\
\text { decisions. The Czech Republic in } 1996\end{array}$ & 34 & 2 & 1998 \\
\hline Vecernik, J. & $\begin{array}{l}\text { COMMUNIS } \\
\text { POST- } \\
\text { COMMUN }\end{array}$ & $\begin{array}{l}\text { The middle class in the Czech reforms: } \\
\text { the interplay between policies and social } \\
\text { stratification }\end{array}$ & 32 & 4 & 1999 \\
\hline Potucek, M. & $\begin{array}{l}\text { J EUR SOC } \\
\text { POLICY }\end{array}$ & $\begin{array}{l}\text { Accession and social policy: the case of } \\
\text { the Czech republic }\end{array}$ & 14 & 3 & 2004 \\
\hline Rehak, J. & SOCIOL CAS & $\begin{array}{l}\text { Quality of data I. Classical model of } \\
\text { measuring reliability and its practical } \\
\text { application }\end{array}$ & 34 & 1 & 1998 \\
\hline
\end{tabular}


Table A5. List of all articles taken into account - listed in alphabetical order (by surnames of authors)

\begin{tabular}{|c|c|c|c|c|c|c|}
\hline Author & Journal & Title of article & Vol. & Nr. & Year & Lang \\
\hline Balon J & $\begin{array}{l}\text { SOCIOL } \\
\text { CAS }\end{array}$ & $\begin{array}{l}\text { Social theory and cultural studies: Two types of } \\
\text { interdisciplinary approach }\end{array}$ & 40 & $\begin{array}{l}1 \\
2\end{array}$ & 2004 & $\mathrm{C}$ \\
\hline Bartova I & $\begin{array}{l}\text { SOCIOL } \\
\text { CAS }\end{array}$ & $\begin{array}{l}\text { Quality of data III. Empirical results of reliability } \\
\text { measurement for selected variables and scales }\end{array}$ & 34 & 3 & 1998 & $\mathrm{C}$ \\
\hline Basl J & $\begin{array}{l}\text { SOCIOL } \\
\text { CAS }\end{array}$ & $\begin{array}{l}\text { Determination of college expectations in OECD } \\
\text { countries: The role of individual and structural } \\
\text { factors }\end{array}$ & 43 & 6 & 2007 & $\mathrm{E}$ \\
\hline Benacek V & $\begin{array}{l}\text { SOCIOL } \\
\text { CAS }\end{array}$ & $\begin{array}{l}\text { The rise of the 'grand entrepreneurs' in the Czech } \\
\text { Republic and their contest for capitalism }\end{array}$ & 42 & 6 & 2006 & $E$ \\
\hline Benacek V & $\begin{array}{l}\text { FINANC } \\
\text { UVER }\end{array}$ & $\begin{array}{l}\text { An authentic private sector in a transition } \\
\text { economy: The roots and alternatives of Czech } \\
\text { capitalism }\end{array}$ & 52 & 3 & 2002 & $\mathrm{C}$ \\
\hline Benacek V & $\begin{array}{l}\text { FINANC } \\
\text { UVER }\end{array}$ & Czech foreign trade flows & 50 & 11 & 2000 & $\mathrm{C}$ \\
\hline Benacek V & POLIT EKON & Foreign direct investment in the Czech economy & 48 & 1 & 2000 & $\mathrm{C}$ \\
\hline Benacek V & $\begin{array}{l}\text { FINANC } \\
\text { UVER }\end{array}$ & $\begin{array}{l}\text { Growth and structural changes in the international } \\
\text { trade in Czech manufacturing industries in 1993- } \\
97\end{array}$ & 49 & 12 & 1999 & $\mathrm{C}$ \\
\hline Bernard J & $\begin{array}{l}\text { SOCIOL } \\
\text { CAS }\end{array}$ & $\begin{array}{l}\text { The social integration of migrants from cities to } \\
\text { villages in the Czech Republic and in Austria }\end{array}$ & 42 & 4 & 2006 & $\mathrm{C}$ \\
\hline Bernard J & $\begin{array}{l}\text { SWS- } \\
\text { RUNDSCHA } \\
U\end{array}$ & $\begin{array}{l}\text { Social integration of immigrants from big-cities-to- } \\
\text { villages in Austria and in the Czech Republic }\end{array}$ & 47 & 2 & 2007 & G \\
\hline Brokl L & $\begin{array}{l}\text { SOCIOL } \\
\text { CAS }\end{array}$ & $\begin{array}{l}\text { The relationship of members of the Czech } \\
\text { parliament to voters as a matter of vertical } \\
\text { accountability }\end{array}$ & 37 & 3 & 2001 & $\mathrm{C}$ \\
\hline Burianek J & $\begin{array}{l}\text { CESK } \\
\text { PSYCHOL }\end{array}$ & $\begin{array}{l}\text { Shall we give up freedom under the pressure of } \\
\text { risks? }\end{array}$ & 47 & 4 & 2003 & $\mathrm{C}$ \\
\hline Burianek J & $\begin{array}{l}\text { JUSTICE } \\
\text { SYST J }\end{array}$ & $\begin{array}{l}\text { The social correlates of punitiveness toward } \\
\text { criminals: A comparison of the Czech Republic } \\
\text { and Florida }\end{array}$ & 23 & 2 & 2002 & $E$ \\
\hline Burianek J & $\begin{array}{l}\text { SOCIOL } \\
\text { CAS }\end{array}$ & $\begin{array}{l}\text { Security risk and its perception in the eyes of the } \\
\text { Czech public }\end{array}$ & 37 & 1 & 2001 & $\mathrm{C}$ \\
\hline Burianek J & $\begin{array}{l}\text { SOCIOLOGI } \\
\text { A }\end{array}$ & $\begin{array}{l}\text { The public and the crime rates in the Czech } \\
\text { Republic: Trends of development. }\end{array}$ & 32 & 1 & 2000 & $\mathrm{~S}$ \\
\hline Cermak D & $\begin{array}{l}\text { SOCIOL } \\
\text { CAS }\end{array}$ & $\begin{array}{l}\text { The influence of territorially specific factors on the } \\
\text { formation of the political attitudes of voters }\end{array}$ & 40 & 4 & 2004 & $\mathrm{C}$ \\
\hline Cermak D & $\begin{array}{l}\text { SOCIOL } \\
\text { CAS }\end{array}$ & $\begin{array}{l}\text { Surveys and aggregate data analysis - A } \\
\text { discussion of the usability of different approaches } \\
\text { in a comparative analysis of political behaviour }\end{array}$ & 39 & 4 & 2003 & $\mathrm{C}$ \\
\hline Cervenka J & $\begin{array}{l}\text { SOTSIOL } \\
\text { ISSLED }\end{array}$ & $\begin{array}{l}\text { Transformation and modernization of Czech } \\
\text { society }\end{array}$ & $?$ & 7 & 2002 & $\mathrm{R}$ \\
\hline Dudova R & $\begin{array}{l}\text { SOCIOL } \\
\text { CAS }\end{array}$ & $\begin{array}{l}\text { Old obligations in the modern world: The father as } \\
\text { provider before and after divorce }\end{array}$ & 42 & 3 & 2006 & $E$ \\
\hline Fafejta M & FILOS CAS & $\begin{array}{l}\text { Dispute and destructive Gemeinschaft (Lyotard, } \\
\text { Richard Sennett) }\end{array}$ & 54 & 4 & 2006 & $\mathrm{C}$ \\
\hline Fafejta M & $\begin{array}{l}\text { SOCIOL } \\
\text { CAS }\end{array}$ & $\begin{array}{l}\text { On some strategies of feminist politics - The } \\
\text { deconstruction of gender symbolism }\end{array}$ & 38 & 5 & 2002 & $\mathrm{C}$ \\
\hline Fafejta M & FILOS CAS & $\begin{array}{l}\text { Sex, gender and feminist politics - An attempt at } \\
\text { deconstructing the concept of naturalness }\end{array}$ & 49 & 5 & 2001 & $\mathrm{C}$ \\
\hline
\end{tabular}




\begin{tabular}{|c|c|c|c|c|c|c|}
\hline Fonadova L & $\begin{array}{l}\text { EUR } \\
\text { SOCIOL } \\
\text { REV }\end{array}$ & $\begin{array}{l}\text { Trends in educational assortative mating in } \\
\text { Central Europe: The Czech Republic, Slovakia, } \\
\text { Poland, and Hungary, 1988-2000 }\end{array}$ & 22 & 3 & 2006 & $E$ \\
\hline Fonadova L & $\begin{array}{l}\text { SOCIOL } \\
\text { CAS }\end{array}$ & $\begin{array}{l}\text { Has the post-communist transformation led to an } \\
\text { increase in educational homogamy in the Czech } \\
\text { Republic after 1989? }\end{array}$ & 40 & 3 & 2004 & $E$ \\
\hline Fric $P$ & $\begin{array}{l}\text { SOCIOL } \\
\text { CAS }\end{array}$ & $\begin{array}{l}\text { A model of Czech society's development and its } \\
\text { modernisation in a global context }\end{array}$ & 40 & 4 & 2004 & C \\
\hline Fric $\mathrm{P}$ & $\begin{array}{l}\text { SOCIOL } \\
\text { CAS }\end{array}$ & $\begin{array}{l}\text { Corruption - deviant behaviour or social } \\
\text { disorganisation? The case of Czech police }\end{array}$ & 37 & 1 & 2001 & C \\
\hline Fucik P & $\begin{array}{l}\text { SOCIOL } \\
\text { CAS }\end{array}$ & $\begin{array}{l}\text { The age homogamy of Czech marriages, 1920- } \\
2000\end{array}$ & 42 & 4 & 2006 & C \\
\hline Gatnar L & $\begin{array}{l}\text { SOTSIOL } \\
\text { ISSLED }\end{array}$ & $\begin{array}{l}\text { Transformation and modernization of Czech } \\
\text { society }\end{array}$ & $?$ & 7 & 2002 & $\mathrm{R}$ \\
\hline Gatnar L & $\begin{array}{l}\text { SOCIOL } \\
\text { CAS }\end{array}$ & $\begin{array}{l}\text { The prospects of the compromise political model: } \\
\text { The results of a post-electoral survey in the Czech } \\
\text { Republic }\end{array}$ & 35 & 3 & 1999 & C \\
\hline Hajek M & $\begin{array}{l}\text { SOCIOL } \\
\text { CAS }\end{array}$ & $\begin{array}{l}\text { The 'Background' and 'Battlefield' in the struggle } \\
\text { for justice: Text analysis of trade union, feminist, } \\
\text { and human rights media communications }\end{array}$ & 42 & 2 & 2006 & C \\
\hline Hamanova J & $\begin{array}{l}\text { SOCIOL } \\
\text { CAS }\end{array}$ & $\begin{array}{l}\text { Quality of data III. Empirical results of reliability } \\
\text { measurement for selected variables and scales }\end{array}$ & 34 & 3 & 1998 & $\mathrm{C}$ \\
\hline Hamar E & $\begin{array}{l}\text { SOCIOL } \\
\text { CAS }\end{array}$ & $\begin{array}{l}\text { Eight women migrants and their shared } \\
\text { transnational world }\end{array}$ & 43 & 1 & 2007 & $S$ \\
\hline Hamplova D & $\begin{array}{l}\text { SOCIOL } \\
\text { CAS }\end{array}$ & $\begin{array}{l}\text { Satisfaction with life, happiness, and family status } \\
\text { in twenty-one European countries }\end{array}$ & 42 & 1 & 2006 & C \\
\hline Hamplova D & $\begin{array}{l}\text { SOCIOL } \\
\text { CAS }\end{array}$ & $\begin{array}{l}\text { Marriage and educational attainment: A dynamic } \\
\text { approach to first union formation }\end{array}$ & 39 & 6 & 2003 & $E$ \\
\hline Hamplova D & $\begin{array}{l}\text { SOCIOL } \\
\text { CAS }\end{array}$ & $\begin{array}{l}\text { Marriage and cohabitation: Qualitative differences } \\
\text { in partnership arrangements }\end{array}$ & 38 & 6 & 2002 & $E$ \\
\hline Hamplova D & $\begin{array}{l}\text { SOTSIOL } \\
\text { ISSLED }\end{array}$ & Religious believers in the Czech republic & $?$ & 7 & 2002 & $\mathrm{R}$ \\
\hline Hamplova D & $\begin{array}{l}\text { SOCIOL } \\
\text { CAS }\end{array}$ & $\begin{array}{l}\text { International Social Survey Program } 1998 \text { - } \\
\text { Religion }\end{array}$ & 36 & 4 & 2000 & C \\
\hline Haskova H & $\begin{array}{l}\text { SOCIOL } \\
\text { CAS }\end{array}$ & $\begin{array}{l}\text { Czech women's civic organising under the state } \\
\text { socialist regime, socio-economic transformation } \\
\text { and the EU accession period }\end{array}$ & 41 & 6 & 2005 & $\mathrm{E}$ \\
\hline Haskova $\mathrm{H}$ & $\begin{array}{l}\text { SOCIOL } \\
\text { CAS }\end{array}$ & $\begin{array}{l}\text { Attitudes of Czech university graduates towards } \\
\text { the position of women in the labour market }\end{array}$ & 36 & 4 & 2000 & C \\
\hline Havelka M & $\begin{array}{l}\text { SOCIOLOGI } \\
\text { A }\end{array}$ & $\begin{array}{l}\text { Czech conservatism and conservative } \\
\text { intellectuals. }\end{array}$ & 34 & 1 & 2002 & $\mathrm{~S}$ \\
\hline Havelka M & $\begin{array}{l}\text { SOCIOLOGI } \\
\text { A }\end{array}$ & $\begin{array}{l}\text { Max Weber and the origins of the sociology of } \\
\text { religion. }\end{array}$ & 30 & 5 & 1998 & S \\
\hline Havlikova J & $\begin{array}{l}\text { SOCIOLOGI } \\
\text { A }\end{array}$ & $\begin{array}{l}\text { The importance of the process of community } \\
\text { planning of social services in addressing the } \\
\text { problem of social exclusion at the local level. }\end{array}$ & 39 & 1 & 2007 & S \\
\hline Hermanova $\mathrm{E}$ & $\begin{array}{l}\text { SOCIOL } \\
\text { CAS }\end{array}$ & $\begin{array}{l}\text { Regional differentiation of the housing market and } \\
\text { its causes }\end{array}$ & 36 & 1 & 2000 & C \\
\hline Hubikova O & $\begin{array}{l}\text { SOCIOLOGI } \\
\text { A }\end{array}$ & $\begin{array}{l}\text { The importance of the process of community } \\
\text { planning of social services in addressing the } \\
\text { problem of social exclusion at the local level. }\end{array}$ & 39 & 1 & 2007 & S \\
\hline Hudeckova H & $\begin{array}{l}\text { SOCIOL } \\
\text { CAS }\end{array}$ & $\begin{array}{l}\text { Rural sociology in education and research at the } \\
\text { Czech University of agriculture in Prague }\end{array}$ & 38 & $\begin{array}{l}1 \\
2\end{array}$ & 2002 & $C$ \\
\hline $\begin{array}{l}\text { Chaloupkova } \\
\mathrm{J}\end{array}$ & $\begin{array}{l}\text { SOCIOL } \\
\text { CAS }\end{array}$ & $\begin{array}{l}\text { Joint or separate income management among } \\
\text { married and cohabitating couples }\end{array}$ & 42 & 5 & 2006 & C \\
\hline
\end{tabular}




\begin{tabular}{|c|c|c|c|c|c|c|}
\hline $\begin{array}{l}\text { Chaloupkova } \\
\mathrm{J}\end{array}$ & $\begin{array}{l}\text { SOCIOL } \\
\text { CAS }\end{array}$ & $\begin{array}{l}\text { Attitudes towards immigrants and the impact of } \\
\text { migration in European countries }\end{array}$ & 42 & 1 & 2006 & $\mathrm{C}$ \\
\hline $\begin{array}{l}\text { Chaloupkova } \\
\mathrm{J}\end{array}$ & $\begin{array}{l}\text { SOCIOL } \\
\text { CAS }\end{array}$ & $\begin{array}{l}\text { Factors influencing the division of housework in } \\
\text { Czech households and evaluations of equitability }\end{array}$ & 41 & 1 & 2005 & $\mathrm{C}$ \\
\hline Illner M & $\begin{array}{l}\text { SOC INDIC } \\
\text { RES }\end{array}$ & $\begin{array}{l}\text { The changing quality of life in a post-communist } \\
\text { country: The case of Czech Republic }\end{array}$ & 43 & $\begin{array}{l}1 \\
2\end{array}$ & 1998 & $E$ \\
\hline Illner M & $\begin{array}{l}\text { SOCIOL } \\
\text { CAS }\end{array}$ & $\begin{array}{l}\text { How regions function - An attempt at evaluating } \\
\text { the performance of regional governments }\end{array}$ & 43 & 5 & 2007 & $\mathrm{C}$ \\
\hline Jerabek H & $\begin{array}{l}\text { SOCIOL } \\
\text { CAS }\end{array}$ & $\begin{array}{l}\text { The measurement of opinion leadership in Czech } \\
\text { sociological research - (The personality strength } \\
\text { scale as a tool for opinion leadership } \\
\text { identification) }\end{array}$ & 39 & 5 & 2003 & $\mathrm{C}$ \\
\hline Jerabek H & $\begin{array}{l}\text { SOCIOL } \\
\text { CAS }\end{array}$ & $\begin{array}{l}\text { The seven weaknesses of Czech sociology - the } \\
\text { personal viewpoint of one sociologist }\end{array}$ & 38 & $\begin{array}{l}1 \\
2\end{array}$ & 2002 & $\mathrm{C}$ \\
\hline Jerabek H & $\begin{array}{l}\text { SOCIOL } \\
\text { CAS }\end{array}$ & $\begin{array}{l}\text { Panel analysis - Method and results of the } \\
\text { research "the people's choice" }\end{array}$ & 36 & 2 & 2000 & $\mathrm{C}$ \\
\hline Jerabek H & $\begin{array}{l}\text { SOCIOL } \\
\text { CAS }\end{array}$ & $\begin{array}{l}\text { The beginnings of P. Lazarsfeld's political } \\
\text { sociology - The people's choice Social } \\
\text { determinants of voter decisions }\end{array}$ & 36 & 1 & 2000 & $\mathrm{C}$ \\
\hline Kabele J & $\begin{array}{l}\text { SOCIOL } \\
\text { CAS }\end{array}$ & $\begin{array}{l}\text { The 'Background' and 'Battlefield' in the struggle } \\
\text { for justice: Text analysis of trade union, feminist, } \\
\text { and human rights media communications }\end{array}$ & 42 & 2 & 2006 & $\mathrm{C}$ \\
\hline Kalvas F & $\begin{array}{l}\text { SOCIOL } \\
\text { CAS }\end{array}$ & $\begin{array}{l}\text { How does the content and structure of television } \\
\text { news affect the perceived importance of issues } \\
\text { among the Czech public? }\end{array}$ & 43 & 2 & 2007 & $\mathrm{C}$ \\
\hline Katrnak T & $\begin{array}{l}\text { EUR } \\
\text { SOCIOL } \\
\text { REV }\end{array}$ & $\begin{array}{l}\text { Trends in educational assortative mating in } \\
\text { Central Europe: The Czech Republic, Slovakia, } \\
\text { Poland, and Hungary, 1988-2000 }\end{array}$ & 22 & 3 & 2006 & $E$ \\
\hline Katrnak T & $\begin{array}{l}\text { SOCIOL } \\
\text { CAS }\end{array}$ & $\begin{array}{l}\text { Has the post-communist transformation led to an } \\
\text { increase in educational homogamy in the Czech } \\
\text { Republic after 1989? }\end{array}$ & 40 & 3 & 2004 & $E$ \\
\hline Katrnak T & $\begin{array}{l}\text { SOCIOLOGI } \\
\text { A }\end{array}$ & $\begin{array}{l}\text { Social and cultural reproduction in theoretical } \\
\text { perspective }\end{array}$ & 35 & 1 & 2003 & $\mathrm{~S}$ \\
\hline Katrnak T & $\begin{array}{l}\text { SOCIOL } \\
\text { CAS }\end{array}$ & $\begin{array}{l}\text { Structural causes of the decline in the number of } \\
\text { marriages and the increase in the number of } \\
\text { single women and men in the Czech Republic in } \\
\text { the 1990s }\end{array}$ & 37 & 2 & 2001 & $\mathrm{C}$ \\
\hline Katrnak T & $\begin{array}{l}\text { SOCIOL } \\
\text { CAS }\end{array}$ & $\begin{array}{l}\text { Modern partner relationships and their } \\
\text { transformation in the age of late modernity }\end{array}$ & 36 & 3 & 2000 & $\mathrm{C}$ \\
\hline Katrnak T & $\begin{array}{l}\text { SOCIOL } \\
\text { CAS }\end{array}$ & $\begin{array}{l}\text { The problem of the relation between religiousness } \\
\text { and anomie }\end{array}$ & 34 & 1 & 1998 & $\mathrm{C}$ \\
\hline Katrnak T & $\begin{array}{l}\text { SOCIOL } \\
\text { CAS }\end{array}$ & $\begin{array}{l}\text { The employed and the unemployed in the Czech } \\
\text { labour market between } 1998 \text { and } 2004\end{array}$ & 43 & 2 & 2007 & $\mathrm{C}$ \\
\hline Keller J & $\begin{array}{l}\text { SOCIOL } \\
\text { CAS }\end{array}$ & Ten subjects for Czech sociology & 38 & $\begin{array}{l}1 \\
2\end{array}$ & 2002 & $\mathrm{C}$ \\
\hline Keller J & $\begin{array}{l}\text { SOTSIOL } \\
\text { ISSLED }\end{array}$ & $\begin{array}{l}\text { The modernization - humanization of society, or } \\
\text { the corrosion of the social being? }\end{array}$ & & 7 & 2002 & $\mathrm{R}$ \\
\hline Kepakova K & \begin{tabular}{|l|}
$\mathrm{SOClOL}$ \\
$\mathrm{CAS}$
\end{tabular} & The sexual behaviour of adolescents and HIV risk & 35 & 2 & 1999 & $\mathrm{C}$ \\
\hline Kolarova M & $\begin{array}{l}\text { SOCIOL } \\
\text { CAS }\end{array}$ & $\begin{array}{l}\text { Gender representation of the anti-globalisation } \\
\text { movement in the alternative media }\end{array}$ & 40 & 6 & 2004 & $\mathrm{E}$ \\
\hline Konopasek Z & HUM STUD & $\begin{array}{l}\text { Political screenings as trials of strength: Making } \\
\text { the communist power/lessness real }\end{array}$ & 29 & 3 & 2006 & $\mathrm{E}$ \\
\hline Konopasek Z & \begin{tabular}{|l} 
SOCIOLOGI \\
A
\end{tabular} & $\begin{array}{l}\text { Construction of communist power and } \\
\text { powerlessness. }\end{array}$ & 31 & 5 & 1999 & $\mathrm{~S}$ \\
\hline
\end{tabular}




\begin{tabular}{|c|c|c|c|c|c|c|}
\hline Konopasek Z & $\begin{array}{l}\text { HIST SOC } \\
\text { RES }\end{array}$ & $\begin{array}{l}\text { Making thinking visible with Atlas.ti: Computer } \\
\text { assisted qualitative analysis as textual practices }\end{array}$ & & & 2007 & $E$ \\
\hline Kostelecky T & $\begin{array}{l}\text { SOCIOL } \\
\text { CAS }\end{array}$ & $\begin{array}{l}\text { The influence of territorially specific factors on the } \\
\text { formation of the political attitudes of voters }\end{array}$ & 40 & 4 & 2004 & $\mathrm{C}$ \\
\hline Kostelecky T & $\begin{array}{l}\text { SOCIOL } \\
\text { CAS }\end{array}$ & $\begin{array}{l}\text { Surveys and aggregate data analysis - A } \\
\text { discussion of the usability of different approaches } \\
\text { in a comparative analysis of political behaviour }\end{array}$ & 39 & 4 & 2003 & $\mathrm{C}$ \\
\hline Kostelecky T & $\begin{array}{l}\text { SOCIOL } \\
\text { CAS }\end{array}$ & $\begin{array}{l}\text { Measuring the performance of national, regional } \\
\text { and local governments }\end{array}$ & 42 & 5 & 2006 & $\mathrm{C}$ \\
\hline Kostelecky T & $\begin{array}{l}\text { SOCIOL } \\
\text { CAS }\end{array}$ & $\begin{array}{l}\text { The Czech population's opinions on the housing } \\
\text { situation and housing policy: Does the Czech } \\
\text { Republic have 'housing classes'? }\end{array}$ & 41 & 2 & 2005 & $\mathrm{C}$ \\
\hline Kostelecky T & $\begin{array}{l}\text { SOCIOL } \\
\text { CAS }\end{array}$ & $\begin{array}{l}\text { A model for predicting election results - An } \\
\text { alternative approach to estimating election results } \\
\text { in electoral districts }\end{array}$ & 41 & 1 & 2005 & $\mathrm{C}$ \\
\hline Kostelecky T & $\begin{array}{l}\text { SOCIOL } \\
\text { CAS }\end{array}$ & $\begin{array}{l}\text { Proposed changes to the electoral law resulting } \\
\text { from amendments to the 'opposition agreement' in } \\
2000 \text { and their possible consequences }\end{array}$ & 36 & 3 & 2000 & $\mathrm{C}$ \\
\hline Kostelecky T & \begin{tabular}{|l|} 
SOCIOL \\
CAS \\
\end{tabular} & $\begin{array}{l}\text { Regional differentiation of the housing market and } \\
\text { its causes }\end{array}$ & 36 & 1 & 2000 & $\mathrm{C}$ \\
\hline Kostelecky T & $\begin{array}{l}\text { SOCIOL } \\
\text { CAS }\end{array}$ & $\begin{array}{l}\text { How regions function - An attempt at evaluating } \\
\text { the performance of regional governments }\end{array}$ & 43 & 5 & 2007 & $\mathrm{C}$ \\
\hline Kreidl M & $\begin{array}{l}\text { EUR } \\
\text { SOCIOL } \\
\text { REV }\end{array}$ & $\begin{array}{l}\text { Trends in educational assortative mating in } \\
\text { Central Europe: The Czech Republic, Slovakia, } \\
\text { Poland, and Hungary, 1988-2000 }\end{array}$ & 22 & 3 & 2006 & $E$ \\
\hline Kreidl M & $\begin{array}{l}\text { SOCIOL } \\
\text { CAS }\end{array}$ & $\begin{array}{l}\text { Has the post-communist transformation led to an } \\
\text { increase in educational homogamy in the Czech } \\
\text { Republic after 1989? }\end{array}$ & 40 & 3 & 2004 & $E$ \\
\hline Kreidl M & $\begin{array}{l}\text { SOCIOL } \\
\text { CAS }\end{array}$ & $\begin{array}{l}\text { Evaluating the influence of survey agencies on the } \\
\text { construct validity of scales }\end{array}$ & 41 & 1 & 2005 & $\mathrm{C}$ \\
\hline Kreidl M & $\begin{array}{l}\text { EUR } \\
\text { SOCIOL } \\
\text { REV }\end{array}$ & $\begin{array}{l}\text { Politics and secondary school tracking in socialist } \\
\text { Czechoslovakia, 1948-1989 }\end{array}$ & 20 & 2 & 2004 & $E$ \\
\hline Kreidl M & $\begin{array}{l}\text { SOCIOL } \\
\text { CAS }\end{array}$ & $\begin{array}{l}\text { Patterns of secondary school tracking in socialist } \\
\text { Czechoslovakia }\end{array}$ & 38 & 5 & 2002 & $\mathrm{C}$ \\
\hline Kreidl M & $\begin{array}{l}\text { SOCIOLOGI } \\
\text { A }\end{array}$ & $\begin{array}{l}\text { Attitudes towards inequalities, stratification } \\
\text { legitimacy, and social experience during transition }\end{array}$ & 33 & 2 & 2001 & $S$ \\
\hline Kreidl M & $\begin{array}{l}\text { SOCIOL } \\
\text { CAS }\end{array}$ & $\begin{array}{l}\text { Changes in status consistency in the Czech } \\
\text { Republic 1991-1999 }\end{array}$ & 35 & 3 & 1999 & $\mathrm{C}$ \\
\hline Kreidl M & $\begin{array}{l}\text { SOCIOL } \\
\text { CAS }\end{array}$ & $\begin{array}{l}\text { The social background of the extreme right in the } \\
\text { Czech Republic }\end{array}$ & 35 & 3 & 1999 & $\mathrm{C}$ \\
\hline Kreidl M & $\begin{array}{l}\text { SOCIOL } \\
\text { CAS }\end{array}$ & $\begin{array}{l}\text { How does the content and structure of television } \\
\text { news affect the perceived importance of issues } \\
\text { among the Czech public? }\end{array}$ & 43 & 2 & 2007 & $\mathrm{C}$ \\
\hline Kreidl M & $\begin{array}{l}\text { SOCIOLOGI } \\
\text { A }\end{array}$ & $\begin{array}{l}\text { School re-entry under socialism: Sponsored } \\
\text { mobility or a second chance? }\end{array}$ & 39 & 2 & 2007 & $S$ \\
\hline Krejci J & $\begin{array}{l}\text { SOCIOL } \\
\text { CAS }\end{array}$ & $\begin{array}{l}\text { International and comparative social science } \\
\text { research and the Czech Republic: A report on } \\
\text { surveys and available data }\end{array}$ & 42 & 1 & 2006 & $\mathrm{C}$ \\
\hline Krejci J & $\begin{array}{l}\text { SOCIOL } \\
\text { CAS }\end{array}$ & Access to Czech social survey data & 38 & 6 & 2002 & $E$ \\
\hline Krejci J & $\begin{array}{l}\text { SOCIOL } \\
\text { CAS }\end{array}$ & Data services for Czech sociology & 38 & $\begin{array}{l}1, \\
2\end{array}$ & 2002 & $\mathrm{C}$ \\
\hline Krejci J & $\begin{array}{l}\text { SOCIOL } \\
\text { CAS }\end{array}$ & $\begin{array}{l}\text { Non-response in probability sample surveys in the } \\
\text { Czech Republic }\end{array}$ & 43 & 3 & 2007 & $\mathrm{E}$ \\
\hline Krizkova A & $\begin{array}{l}\text { SOCIOL } \\
\text { CAS }\end{array}$ & $\begin{array}{l}\text { The career patterns of women in management - } \\
\text { The strategies of women within the gender regime } \\
\text { of organisation }\end{array}$ & 39 & 4 & 2003 & $\mathrm{C}$ \\
\hline
\end{tabular}




\begin{tabular}{|c|c|c|c|c|c|c|}
\hline Kubatova H & $\begin{array}{l}\text { SOCIOLOGI } \\
\text { A }\end{array}$ & $\begin{array}{l}\text { Cultural transformation of the Czech society } \\
\text { against the background of its economic and } \\
\text { political transformation }\end{array}$ & 37 & 4 & 2005 & $\mathrm{~S}$ \\
\hline Kucerova E & $\begin{array}{l}\text { SOCIOL } \\
\text { RURALIS }\end{array}$ & $\begin{array}{l}\text { The project class in Central Europe: The Czech } \\
\text { and Hungarian cases }\end{array}$ & 46 & 1 & 2006 & $E$ \\
\hline Kucerova E & $\begin{array}{l}\text { PERCEPT } \\
\text { MOTOR } \\
\text { SKILL }\end{array}$ & $\begin{array}{l}\text { Sex of expresser and correct perception of facial } \\
\text { expressions of emotion }\end{array}$ & 104 & 3 & 2007 & $E$ \\
\hline Kuchar P & $\begin{array}{l}\text { SOTSIOL } \\
\text { ISSLED }\end{array}$ & $\begin{array}{l}\text { Transformation and modernization of Czech } \\
\text { society }\end{array}$ & $?$ & 7 & 2002 & $\mathrm{R}$ \\
\hline Kuchar P & $\begin{array}{l}\text { J EUR SOC } \\
\text { POLICY }\end{array}$ & $\begin{array}{l}\text { Social consequences of unemployment: an East- } \\
\text { West comparison }\end{array}$ & 11 & 1 & 2001 & $E$ \\
\hline Kuchar P & $\begin{array}{l}\text { WORK } \\
\text { EMPLOY } \\
\text { SOC }\end{array}$ & $\begin{array}{l}\text { Employment experience and organisational } \\
\text { commitment: An East-West European comparison }\end{array}$ & 13 & 4 & 1999 & $E$ \\
\hline Lebeda T & $\begin{array}{l}\text { SOCIOL } \\
\text { CAS }\end{array}$ & $\begin{array}{l}\text { The proportionality of electoral formulas for } \\
\text { systems of proportional representation }\end{array}$ & 42 & 5 & 2006 & $\mathrm{C}$ \\
\hline Lebeda T & $\begin{array}{l}\text { SOCIOL } \\
\text { CAS }\end{array}$ & $\begin{array}{l}\text { Real quota theory, an alternative approach to } \\
\text { measuring electoral proportionality }\end{array}$ & 42 & 4 & 2006 & $\mathrm{C}$ \\
\hline Lebeda T & $\begin{array}{l}\text { OSTEUROP } \\
\text { A }\end{array}$ & $\begin{array}{l}\text { Stalemate in Central Europe - The } 2006 \text { Czech } \\
\text { parlimentary election }\end{array}$ & 56 & 7 & 2006 & $\mathrm{G}$ \\
\hline Lebeda T & $\begin{array}{l}\text { SOCIOL } \\
\text { CAS }\end{array}$ & $\begin{array}{l}\text { Active citizenship and satisfaction with democracy } \\
\text { in Europe }\end{array}$ & 42 & 1 & 2006 & $\mathrm{C}$ \\
\hline Lebeda T & $\begin{array}{l}\text { SOCIOL } \\
\text { CAS }\end{array}$ & $\begin{array}{l}\text { The main variables of systems of proportional } \\
\text { representation }\end{array}$ & 37 & 4 & 2001 & $\mathrm{C}$ \\
\hline Linek L & $\begin{array}{l}\text { SOCIOL } \\
\text { CAS }\end{array}$ & $\begin{array}{l}\text { Why Czech parliamentary party groups vote less } \\
\text { unitedly. The role of frequent voting and big } \\
\text { majorities in passing bills }\end{array}$ & 41 & 3 & 2005 & $E$ \\
\hline Linek L & $\begin{array}{l}\text { SOCIOL } \\
\text { CAS }\end{array}$ & $\begin{array}{l}\text { Surveys of MPs and Senators in the parliament of } \\
\text { the Czech Republic between } 1993 \text { and } 2005\end{array}$ & 41 & 3 & 2005 & $E$ \\
\hline Lostak M & $\begin{array}{l}\text { SOCIOL } \\
\text { CAS }\end{array}$ & $\begin{array}{l}\text { Rural sociology in education and research at the } \\
\text { Czech University of agriculture in Prague }\end{array}$ & 38 & $\begin{array}{l}1, \\
2\end{array}$ & 2002 & $\mathrm{C}$ \\
\hline Louckova I & $\begin{array}{l}\text { SOCIOL } \\
\text { CAS }\end{array}$ & $\begin{array}{l}\text { Towards integrated strategies in social work } \\
\text { research and beyond }\end{array}$ & 37 & 3 & 2001 & $\mathrm{C}$ \\
\hline Louzek M & $\begin{array}{l}\text { SOCIOLOGI } \\
\text { A }\end{array}$ & $\begin{array}{l}\text { The economic sociology of Joseph A. } \\
\text { Schumpeter. }\end{array}$ & 39 & 1 & 2007 & $S$ \\
\hline Lux M & $\begin{array}{l}\text { SOCIOL } \\
\text { CAS }\end{array}$ & $\begin{array}{l}\text { Housing affordability of rental and owner-occupied } \\
\text { housing over the course of the economic } \\
\text { transformation in the Czech Republic (1991-2003) }\end{array}$ & 42 & 5 & 2006 & $\mathrm{C}$ \\
\hline Lux M & $\begin{array}{l}\text { SOCIOL } \\
\text { CAS }\end{array}$ & On housing satisfaction among Czech citizens & 41 & 2 & 2005 & $\mathrm{C}$ \\
\hline Lux M & $\begin{array}{l}\text { FINANC } \\
\text { UVER }\end{array}$ & Equilibrium rent and housing policy implications & 53 & $\begin{array}{l}1, \\
2\end{array}$ & 2003 & $\mathrm{C}$ \\
\hline Lux M & $\begin{array}{l}\text { SOCIOL } \\
\text { CAS }\end{array}$ & $\begin{array}{l}\text { Social housing in the Czech Republic in a } \\
\text { comparative context }\end{array}$ & 36 & 2 & 2000 & $\mathrm{C}$ \\
\hline Lux M & $\begin{array}{l}\text { SOCIOL } \\
\text { CAS }\end{array}$ & $\begin{array}{l}\text { The effect of housing conditions on the intended } \\
\text { labour migration of the Czech population }\end{array}$ & 43 & 2 & 2007 & $\mathrm{C}$ \\
\hline Lux M & $\begin{array}{l}\text { URBAN } \\
\text { STUD }\end{array}$ & $\begin{array}{l}\text { The quasi-normative approach to housing } \\
\text { affordability: The case of the Czech Republic }\end{array}$ & 44 & $\begin{array}{l}5, \\
6\end{array}$ & 2007 & $\mathrm{E}$ \\
\hline Lyons $\mathrm{P}$ & $\begin{array}{l}\text { POLIT } \\
\text { STUD- } \\
\text { LONDON }\end{array}$ & $\begin{array}{l}\text { Pragmatists, ideologues and the general law of } \\
\text { curvilinear disparity: The case of the Irish Labour } \\
\text { Party }\end{array}$ & 54 & 4 & 2006 & $\mathrm{E}$ \\
\hline Lyons $\mathrm{P}$ & $\begin{array}{l}\text { SOCIOL } \\
\text { CAS }\end{array}$ & $\begin{array}{l}\text { 'It's the economy, stupid' - Popular support for EU } \\
\text { accession in the Czech Republic }\end{array}$ & 43 & 3 & 2007 & $\mathrm{E}$ \\
\hline Manea BEC & $\begin{array}{l}\text { SOCIOL } \\
\text { CAS }\end{array}$ & $\begin{array}{l}\text { One-child families in the Czech republic. Who are } \\
\text { the people who have or plan to have just one } \\
\text { child? }\end{array}$ & 43 & 4 & 2007 & $\mathrm{C}$ \\
\hline
\end{tabular}




\begin{tabular}{|c|c|c|c|c|c|c|}
\hline $\begin{array}{l}\text { Mansfeldova } \\
\text { Z }\end{array}$ & $\begin{array}{l}\text { SOCIOL } \\
\text { CAS }\end{array}$ & $\begin{array}{l}\text { The relationship of members of the Czech } \\
\text { parliament to voters as a matter of vertical } \\
\text { accountability }\end{array}$ & 37 & 3 & 2001 & $\mathrm{C}$ \\
\hline $\begin{array}{l}\text { Mansfeldova } \\
\text { Z }\end{array}$ & $\begin{array}{l}\text { SOCIOL } \\
\text { CAS }\end{array}$ & $\begin{array}{l}\text { Executive-legislative relations in the budgeting } \\
\text { process in the Czech Republic }\end{array}$ & 41 & 3 & 2005 & $E$ \\
\hline Marada M & $\begin{array}{l}\text { SOCIOL } \\
\text { CAS }\end{array}$ & Ethos, interests, dispositions & 36 & 4 & 2000 & $\mathrm{C}$ \\
\hline Mares P & $\begin{array}{l}\text { SOCIOL } \\
\text { CAS }\end{array}$ & $\begin{array}{l}\text { Poverty, deprivation and social exlusion: The } \\
\text { unemployed and the working poor }\end{array}$ & 42 & 4 & 2006 & $\mathrm{C}$ \\
\hline Mares P & $\begin{array}{l}\text { SOC } \\
\text { POLICY } \\
\text { ADMIN }\end{array}$ & $\begin{array}{l}\text { Poverty, social exclusion and social policy in the } \\
\text { Czech Republic }\end{array}$ & 40 & 3 & 2006 & $E$ \\
\hline Mares P & $\begin{array}{l}\text { FINANC } \\
\text { UVER }\end{array}$ & $\begin{array}{l}\text { Unemployment, labour marginalisation and } \\
\text { deprivation }\end{array}$ & 55 & $\begin{array}{l}1 \\
2\end{array}$ & 2005 & $E$ \\
\hline Mares P & $\begin{array}{l}\text { SOCIOL } \\
\text { CAS }\end{array}$ & $\begin{array}{l}\text { From work as an emancipator to work that's no } \\
\text { longer there }\end{array}$ & 40 & $\begin{array}{l}1, \\
2\end{array}$ & 2004 & $\mathrm{C}$ \\
\hline Mares P & $\begin{array}{l}\text { SOCIOL } \\
\text { CAS }\end{array}$ & $\begin{array}{l}\text { The long-term unemployed - Living conditions and } \\
\text { strategies }\end{array}$ & 39 & 1 & 2003 & $\mathrm{C}$ \\
\hline Mares $\mathrm{P}$ & $\begin{array}{l}\text { SOCIOL } \\
\text { CAS }\end{array}$ & Poverty, marginalisation, social exclusion & 36 & 3 & 2000 & $\mathrm{C}$ \\
\hline Mares P & $\begin{array}{l}\text { SOCIOL } \\
\text { CAS }\end{array}$ & $\begin{array}{l}\text { Failure of social policy: Non-take-up of social } \\
\text { security as a problem of social cognisance }\end{array}$ & 36 & 2 & 2000 & $\mathrm{C}$ \\
\hline Mares $\mathrm{P}$ & $\begin{array}{l}\text { SOCIOLOGI } \\
\text { A }\end{array}$ & $\begin{array}{l}\text { Experience of the Czech population with the } \\
\text { labour market. }\end{array}$ & 31 & 5 & 1999 & $\mathrm{~S}$ \\
\hline Mares P & $\begin{array}{l}\text { SOCIOL } \\
\text { CAS }\end{array}$ & Ageing and politics & 34 & 3 & 1998 & $\mathrm{C}$ \\
\hline Mares P & $\begin{array}{l}\text { SOCIOL } \\
\text { CAS }\end{array}$ & $\begin{array}{l}\text { The employed and the unemployed in the Czech } \\
\text { labour market between } 1998 \text { and } 2004\end{array}$ & 43 & 2 & 2007 & $\mathrm{C}$ \\
\hline Mateju P & $\begin{array}{l}\text { SOCIOL } \\
\text { CAS }\end{array}$ & $\begin{array}{l}\text { Changes in status consistency in the Czech } \\
\text { Republic 1991-1999 }\end{array}$ & 35 & 3 & 1999 & $\mathrm{C}$ \\
\hline Mateju P & $\begin{array}{l}\text { SOCIOL } \\
\text { CAS }\end{array}$ & $\begin{array}{l}\text { Interpersonal trust and mutually beneficial } \\
\text { exchanges: Measuring social capital for } \\
\text { comparative analyses }\end{array}$ & 42 & 3 & 2006 & $\mathrm{E}$ \\
\hline Mateju P & $\begin{array}{l}\text { SOCIOL } \\
\text { CAS }\end{array}$ & $\begin{array}{l}\text { The roots of the socio-psychological model of } \\
\text { social stratification }\end{array}$ & 41 & 1 & 2005 & $\mathrm{C}$ \\
\hline Mateju P & $\begin{array}{l}\text { BRIT J } \\
\text { SOCIOL } \\
\text { EDUC }\end{array}$ & $\begin{array}{l}\text { The role of the family and the school in the } \\
\text { reproduction of educational inequalities in the } \\
\text { post-Communist Czech Republic }\end{array}$ & 26 & 1 & 2005 & $E$ \\
\hline Mateju P & $\begin{array}{l}\text { SOCIOL } \\
\text { CAS }\end{array}$ & $\begin{array}{l}\text { The role of the family and the school in the } \\
\text { reproduction of educational inequalities - A } \\
\text { sociological look at the role of multi-year gymnasia } \\
\text { in the context of the results of the PISA } 2000 \\
\text { study }\end{array}$ & 39 & 5 & 2003 & $\mathrm{C}$ \\
\hline Mateju P & $\begin{array}{l}\text { SOCIOL } \\
\text { CAS }\end{array}$ & $\begin{array}{l}\text { Transition to university under communism and } \\
\text { after its demise - The role of socio-economic } \\
\text { background in the transition between secondary } \\
\text { and tertiary education in the Czech Republic } \\
1948-1998\end{array}$ & 39 & 3 & 2003 & $\mathrm{E}$ \\
\hline Mateju P & $\begin{array}{l}\text { SOCIOL } \\
\text { CAS }\end{array}$ & Czech higher education still at the crossroads & 39 & 3 & 2003 & $E$ \\
\hline Mateju P & $\begin{array}{l}\text { SOCIOLOGI } \\
\text { A }\end{array}$ & $\begin{array}{l}\text { Cultural and socio-economic origins of unequal } \\
\text { opportunities in attaining higher education in the } \\
\text { Czech Republic (1948-1999). }\end{array}$ & 36 & 1 & 2004 & $\mathrm{~S}$ \\
\hline Mateju P & $\begin{array}{l}\text { INT J COMP } \\
\text { SOCIOL }\end{array}$ & $\begin{array}{l}\text { Who votes left after the fall of communism? The } \\
\text { Czech republic in comparative perspective }\end{array}$ & 40 & 1 & 1999 & $\mathrm{E}$ \\
\hline Mateju P & $\begin{array}{l}\text { SOCIOL } \\
\text { CAS }\end{array}$ & $\begin{array}{l}\text { The crystallisation of political attitudes and the } \\
\text { political spectrum in the Czech Republic }\end{array}$ & 34 & 2 & 1998 & $\mathrm{C}$ \\
\hline Mateju P & $\begin{array}{l}\text { SOCIOL } \\
\text { CAS }\end{array}$ & $\begin{array}{l}\text { The role of politically relevant attitudes and value } \\
\text { orientations in electoral decisions. The Czech }\end{array}$ & 34 & 2 & 1998 & C \\
\hline
\end{tabular}




\begin{tabular}{|c|c|c|c|c|c|c|}
\hline & & Republic in 1996 & & & & \\
\hline Mateju P & $\begin{array}{l}\text { COMMUNIS } \\
\text { POST- } \\
\text { COMMUN }\end{array}$ & $\begin{array}{l}\text { Values and electoral decisions in the Czech } \\
\text { Republic }\end{array}$ & 31 & 3 & 1998 & $\mathrm{E}$ \\
\hline Mateju P & $\begin{array}{l}\text { SOCIOL } \\
\text { CAS }\end{array}$ & $\begin{array}{l}\text { Determination of college expectations in OECD } \\
\text { countries: The role of individual and structural } \\
\text { factors }\end{array}$ & 43 & 6 & 2007 & $\mathrm{E}$ \\
\hline Mozny I & $\begin{array}{l}\text { SOCIOL } \\
\text { CAS }\end{array}$ & The Brno anomaly? Sociology in Brno 1963-1989 & 40 & 5 & 2004 & $\mathrm{C}$ \\
\hline Mozny I & $\begin{array}{l}\text { SOCIOL } \\
\text { CAS }\end{array}$ & Old age and the disconnected course of life & 34 & 3 & 1998 & $\mathrm{C}$ \\
\hline Muller K & $\begin{array}{l}\text { SOTSIOL } \\
\text { ISSLED }\end{array}$ & $\begin{array}{l}\text { Transformation and modernization of Czech } \\
\text { society }\end{array}$ & $?$ & 7 & 2002 & $\mathrm{R}$ \\
\hline Muller K & $\begin{array}{l}\text { SOCIOL } \\
\text { CAS }\end{array}$ & $\begin{array}{l}\text { The civil society concept: An attempt at a } \\
\text { complementary approach. Tocqueville's legacy } \\
\text { and Giddens' perspective }\end{array}$ & 39 & 5 & 2003 & $\mathrm{C}$ \\
\hline Musil J & $\begin{array}{l}\text { SOCIOL } \\
\text { CAS }\end{array}$ & How the sociology of housing emerged & 41 & 2 & 2005 & $\mathrm{C}$ \\
\hline Musil J & $\begin{array}{l}\text { SOCIOL } \\
\text { CAS }\end{array}$ & $\begin{array}{l}\text { Comments on Czech sociology under the } \\
\text { communist regime }\end{array}$ & 40 & 5 & 2004 & $\mathrm{C}$ \\
\hline Musil J & ESPRIT & $\begin{array}{l}\text { The expansion of the European Union and the } \\
\text { question of the Sudeten Germans }\end{array}$ & & 11 & 2002 & $\mathrm{~F}$ \\
\hline Musil J & $\begin{array}{l}\text { SOCIOL } \\
\text { CAS }\end{array}$ & $\begin{array}{l}\text { Some comments on contemporary Czech } \\
\text { sociology }\end{array}$ & 38 & $\begin{array}{l}1 \\
2\end{array}$ & 2002 & $\mathrm{C}$ \\
\hline Musil J & $\begin{array}{l}\text { SOCIOL } \\
\text { CAS }\end{array}$ & $\begin{array}{l}\text { Urban development and planning in central } \\
\text { Europe under communist regimes - The } \\
\text { perspective of historical sociology }\end{array}$ & 37 & 3 & 2001 & $\mathrm{C}$ \\
\hline Musil J & $\begin{array}{l}\text { SOCIOL } \\
\text { CAS }\end{array}$ & $\begin{array}{l}\text { Masaryk's social question, socialism, and } \\
\text { contemporary communitarianism }\end{array}$ & 34 & 4 & 1998 & $\mathrm{C}$ \\
\hline Musil J & $\begin{array}{l}\text { SOCIOL } \\
\text { CAS }\end{array}$ & Patocka's Europe and the Czechs & 43 & 4 & 2007 & $\mathrm{C}$ \\
\hline Musil L & $\begin{array}{l}\text { SOCIOLOGI } \\
\text { A }\end{array}$ & $\begin{array}{l}\text { The importance of micro - Social organisation for } \\
\text { working of the welfare state. }\end{array}$ & 32 & 1 & 2000 & $\mathrm{~S}$ \\
\hline Navratil P & $\begin{array}{l}\text { SOCIOL } \\
\text { CAS }\end{array}$ & Social work as a social construction & 34 & 1 & 1998 & $\mathrm{C}$ \\
\hline $\begin{array}{l}\text { Nedbalkova } \\
\mathrm{K}\end{array}$ & $\begin{array}{l}\text { SOCIOL } \\
\text { CAS }\end{array}$ & $\begin{array}{l}\text { Does prison have a gender? Masculinity and } \\
\text { femininity in prison subcultures }\end{array}$ & 39 & 4 & 2003 & $\mathrm{C}$ \\
\hline $\begin{array}{l}\text { Nedbalkova } \\
\mathrm{K}\end{array}$ & $\begin{array}{l}\text { SOCIOL } \\
\text { CAS }\end{array}$ & Gay subculture in Brno & 36 & 3 & 2000 & $\mathrm{C}$ \\
\hline Nekola M & $\begin{array}{l}\text { SOCIOL } \\
\text { CAS }\end{array}$ & $\begin{array}{l}\text { The Czech economic elite after fifteen years of } \\
\text { post-socialist transformation }\end{array}$ & 42 & 3 & 2006 & $\mathrm{E}$ \\
\hline Nespor ZR & $\begin{array}{l}\text { SOCIOL } \\
\text { CAS }\end{array}$ & $\begin{array}{l}\text { 'The son has ploughed', but a foreign son. Five } \\
\text { case studies on transformation strategies in } \\
\text { Czech agriculture after } 1989\end{array}$ & 42 & 6 & 2006 & $\mathrm{E}$ \\
\hline Nespor ZR & HIST JAHR & $\begin{array}{l}\text { Nation instead of confession. The decline of } \\
\text { confessional perception patterns and the accrual } \\
\text { of patriotism in Bohemia at the turning point of the } \\
\text { 18th to the 19th century }\end{array}$ & 126 & & 2006 & G \\
\hline Nespor ZR & $\begin{array}{l}\text { SOCIOL } \\
\text { CAS }\end{array}$ & $\begin{array}{l}\text { The re-emigration of Czech western emigrants in } \\
\text { the } 1990 \text { s from the perspective of economic } \\
\text { sociology }\end{array}$ & 41 & 1 & 2005 & $\mathrm{C}$ \\
\hline Nespor ZR & $\begin{array}{l}\text { SOCIOL } \\
\text { CAS }\end{array}$ & Two traditions of Czech sociology of religion & 40 & 4 & 2004 & $\mathrm{C}$ \\
\hline Nespor ZR & $\begin{array}{l}\text { SOCIOL } \\
\text { CAS }\end{array}$ & $\begin{array}{l}\text { Czech folk music in the } 1960 \text { s- } 80 \text { s from the point } \\
\text { of view of the sociology of religion }\end{array}$ & 39 & 1 & 2003 & $\mathrm{C}$ \\
\hline Nespor ZR & $\begin{array}{l}\text { SOCIOL } \\
\text { CAS }\end{array}$ & $\begin{array}{l}\text { Religious processes in contemporary Czech } \\
\text { society }\end{array}$ & 40 & 3 & 2004 & $\mathrm{E}$ \\
\hline
\end{tabular}




\begin{tabular}{|c|c|c|c|c|c|c|}
\hline Nespor ZR & $\begin{array}{l}\text { SOCIOL } \\
\text { CAS }\end{array}$ & $\begin{array}{l}\text { The disappointed and disgruntled: A study of the } \\
\text { return in the 1990s of Czech emigrants from the } \\
\text { communist era }\end{array}$ & 38 & 6 & 2002 & $E$ \\
\hline Nespor ZR & $\begin{array}{l}\text { SOCIOL } \\
\text { CAS }\end{array}$ & $\begin{array}{l}\text { The issue of the subjective justification of actions } \\
\text { in the works of Max Weber, Clifford Geertz and } \\
\text { Rudolf Bultmann }\end{array}$ & 38 & 5 & 2002 & $\mathrm{C}$ \\
\hline Nespor ZR & $\begin{array}{l}\text { SOCIOL } \\
\text { CAS }\end{array}$ & $\begin{array}{l}\text { Czech sociology of religion in the period between } \\
1948 \text { and } 1989\end{array}$ & 43 & 4 & 2007 & $\mathrm{C}$ \\
\hline Nespor ZR & $\begin{array}{l}\text { SOCIOL } \\
\text { CAS }\end{array}$ & $\begin{array}{l}\text { Three-quarters of a century ago: A quantified } \\
\text { essay on Czech sociological journals in the pre- } \\
\text { Marxist era and today }\end{array}$ & 43 & 2 & 2007 & $\mathrm{C}$ \\
\hline Nesporova O & $\begin{array}{l}\text { SOCIOL } \\
\text { CAS }\end{array}$ & $\begin{array}{l}\text { Believer perspectives on death and funeral } \\
\text { practices in a non-believing country }\end{array}$ & 43 & 6 & 2007 & $E$ \\
\hline Novak M & $\begin{array}{l}\text { SOCIOL } \\
\text { CAS }\end{array}$ & $\begin{array}{l}\text { Between the primacy of politics and economic } \\
\text { development: Raymond Aron's sociological } \\
\text { conception of industrial societies }\end{array}$ & 41 & 5 & 2005 & $\mathrm{C}$ \\
\hline Novak M & $\begin{array}{l}\text { SOCIOL } \\
\text { CAS }\end{array}$ & $\begin{array}{l}\text { Popper versus Schumpeter: A comparison of two } \\
\text { non-classical theories of democracy }\end{array}$ & 39 & 1 & 2003 & $\mathrm{C}$ \\
\hline Novak M & $\begin{array}{l}\text { SOCIOL } \\
\text { CAS }\end{array}$ & $\begin{array}{l}\text { Aristotle's political sociology and modern } \\
\text { representative democracy }\end{array}$ & 37 & 4 & 2001 & $\mathrm{C}$ \\
\hline Novak M & \begin{tabular}{|l|} 
SOCIOL \\
CAS
\end{tabular} & s there one best "model" of democracy? (part two) & 34 & 2 & 1998 & $\mathrm{C}$ \\
\hline Novak M & $\begin{array}{l}\text { SOCIOL } \\
\text { CAS }\end{array}$ & Is there one best 'model' of democracy? (first part) & 34 & 1 & 1998 & $\mathrm{C}$ \\
\hline Patockova V & $\begin{array}{l}\text { SOCIOL } \\
\text { CAS }\end{array}$ & $\begin{array}{l}\text { Measuring the performance of national, regional } \\
\text { and local governments }\end{array}$ & 42 & 5 & 2006 & $\mathrm{C}$ \\
\hline Patockova V & $\begin{array}{l}\text { SOCIOL } \\
\text { CAS }\end{array}$ & $\begin{array}{l}\text { How regions function - An attempt at evaluating } \\
\text { the performance of regional governments }\end{array}$ & 43 & 5 & 2007 & $\mathrm{C}$ \\
\hline Petrusek M & $\begin{array}{l}\text { SOCIOL } \\
\text { CAS }\end{array}$ & $\begin{array}{l}\text { Raymond Aron (1905-2005): The } \\
\text { phenomenologist and analyst of freedom and evil }\end{array}$ & 41 & 5 & 2005 & $\mathrm{C}$ \\
\hline Petrusek M & $\begin{array}{l}\text { SOCIOL } \\
\text { CAS }\end{array}$ & $\begin{array}{l}\text { Teaching sociology in the periods before and } \\
\text { during normalisation (1964-1989) - (The } \\
\text { relationship between knowledge and power, } \\
\text { science and politics, and reality and myth) }\end{array}$ & 40 & 5 & 2004 & $\mathrm{C}$ \\
\hline Petrusek M & $\begin{array}{l}\text { SOCIOL } \\
\text { CAS }\end{array}$ & $\begin{array}{l}\text { A century of extremes and Kitsch. The evolution } \\
\text { and transformation of the sociology of culture and } \\
\text { art in the 20th century }\end{array}$ & 40 & $\begin{array}{l}1 \\
2\end{array}$ & 2004 & $\mathrm{C}$ \\
\hline Petrusek M & \begin{tabular}{|l|} 
SOCIOL \\
CAS \\
\end{tabular} & $\begin{array}{l}\text { A lesson from the crisis-free evolution of Czech } \\
\text { sociology }\end{array}$ & 38 & $\begin{array}{l}1 \\
2\end{array}$ & 2002 & $\mathrm{C}$ \\
\hline Potucek M & $\begin{array}{l}\text { SOCIOL } \\
\text { CAS }\end{array}$ & $\begin{array}{l}\text { A model of Czech society's development and its } \\
\text { modernisation in a global context }\end{array}$ & 40 & 4 & 2004 & $\mathrm{C}$ \\
\hline Potucek M & $\begin{array}{l}\text { J EUR SOC } \\
\text { POLICY }\end{array}$ & $\begin{array}{l}\text { Accession and social policy: the case of the } \\
\text { Czech republic }\end{array}$ & 14 & 3 & 2004 & $\mathrm{E}$ \\
\hline Potucek M & $\begin{array}{l}\text { REV ETUD } \\
\text { COMP EST- } \\
\text { O }\end{array}$ & Czech social policy in theory and practice & 29 & 3 & 1998 & $\mathrm{~F}$ \\
\hline Pridalova M & \begin{tabular}{|l|} 
SOCIOL \\
CAS \\
\end{tabular} & Why is death taboo in modern times? & 34 & 3 & 1998 & $\mathrm{C}$ \\
\hline Prusa L & $\begin{array}{l}\text { FINANC } \\
\text { UVER }\end{array}$ & $\begin{array}{l}\text { Social security and the tax system in the CR: } \\
\text { Recent history and outlook }\end{array}$ & 51 & 12 & 2001 & $\mathrm{C}$ \\
\hline Rabusic L & $\begin{array}{l}\text { SOCIOL } \\
\text { CAS }\end{array}$ & $\begin{array}{l}\text { Adult education in the Czech Republic - Who } \\
\text { participates and why }\end{array}$ & 42 & 6 & 2006 & $\mathrm{E}$ \\
\hline Rabusic L & $\begin{array}{l}\text { SOCIOL } \\
\text { CAS }\end{array}$ & $\begin{array}{l}\text { Why are they all so eager to retire? (On the } \\
\text { transition to retirement in the Czech Republic) }\end{array}$ & 40 & 3 & 2004 & $E$ \\
\hline Rabusic L & $\begin{array}{l}\text { SOCIOL } \\
\text { CAS }\end{array}$ & Is Czech society 'post-materialist'? & 36 & 1 & 2000 & $\mathrm{C}$ \\
\hline Rabusic L & $\begin{array}{l}\text { SOCIOL } \\
\text { CAS }\end{array}$ & The Czech welfare state and its legitimacy & 35 & 4 & 1999 & $\mathrm{C}$ \\
\hline
\end{tabular}




\begin{tabular}{|c|c|c|c|c|c|c|}
\hline Rabusic L & $\begin{array}{l}\text { SOCIOL } \\
\text { CAS }\end{array}$ & $\begin{array}{l}\text { Welfare state solidarity and support. The Czech } \\
\text { Republic and the Netherlands in comparison }\end{array}$ & 35 & 3 & 1999 & $\mathrm{C}$ \\
\hline Rabusic L & $\begin{array}{l}\text { SOCIOL } \\
\text { CAS }\end{array}$ & The sexual behaviour of adolescents and HIV risk & 35 & 2 & 1999 & $\mathrm{C}$ \\
\hline Rabusic L & $\begin{array}{l}\text { SOCIOL } \\
\text { CAS }\end{array}$ & Temporal aspects of the Czech retirement age & 34 & 3 & 1998 & $\mathrm{C}$ \\
\hline Rabusic L & $\begin{array}{l}\text { SOCIOL } \\
\text { CAS }\end{array}$ & Are the Czech elderly poor? & 34 & 3 & 1998 & $\mathrm{C}$ \\
\hline Rabusic L & $\begin{array}{l}\text { SOCIOL } \\
\text { CAS }\end{array}$ & $\begin{array}{l}\text { One-child families in the Czech republic. Who are } \\
\text { the people who have or plan to have just one } \\
\text { child? }\end{array}$ & 43 & 4 & 2007 & $\mathrm{C}$ \\
\hline Rabusic L & $\begin{array}{l}\text { SOCIOL } \\
\text { CAS }\end{array}$ & $\begin{array}{l}\text { Some notes on the obsession of the Czech social } \\
\text { sciences with statistical significance }\end{array}$ & 43 & 2 & 2007 & $\mathrm{C}$ \\
\hline $\begin{array}{l}\text { Rabusicova } \\
\text { M }\end{array}$ & $\begin{array}{l}\text { SOCIOL } \\
\text { CAS }\end{array}$ & $\begin{array}{l}\text { Adult education in the Czech Republic - Who } \\
\text { participates and why }\end{array}$ & 42 & 6 & 2006 & $E$ \\
\hline Radimska R & $\begin{array}{l}\text { SOCIOL } \\
\text { CAS }\end{array}$ & $\begin{array}{l}\text { Verification of indicators used in research of } \\
\text { opinions on the crime-rate }\end{array}$ & 37 & 1 & 2001 & $\mathrm{C}$ \\
\hline Radimska R & $\begin{array}{l}\text { SOCIOL } \\
\text { CAS }\end{array}$ & $\begin{array}{l}\text { The individual and the family: The theory of } \\
\text { private life according to Franqois de Singly }\end{array}$ & 39 & 5 & 2003 & $\mathrm{C}$ \\
\hline $\begin{array}{l}\text { Rakusanova } \\
\text { P }\end{array}$ & $\begin{array}{l}\text { SOCIOL } \\
\text { CAS }\end{array}$ & $\begin{array}{l}\text { Why Czech parliamentary party groups vote less } \\
\text { unitedly. The role of frequent voting and big } \\
\text { majorities in passing bills }\end{array}$ & 41 & 3 & 2005 & $E$ \\
\hline Rasek A & $\begin{array}{l}\text { SOCIOL } \\
\text { CAS }\end{array}$ & $\begin{array}{l}\text { Sociology at the workplace in the normalisation } \\
\text { period: The SEPIMO methodology }\end{array}$ & 40 & 5 & 2004 & $\mathrm{C}$ \\
\hline Rehak J & $\begin{array}{l}\text { SOCIOL } \\
\text { CAS }\end{array}$ & $\begin{array}{l}\text { Quality of data II. Approaches to the evaluation of } \\
\text { research instruments based on covariance } \\
\text { structure modelling }\end{array}$ & 34 & 2 & 1998 & $\mathrm{C}$ \\
\hline Rehak J & $\begin{array}{l}\text { SOCIOL } \\
\text { CAS }\end{array}$ & $\begin{array}{l}\text { Quality of data III. Empirical results of reliability } \\
\text { measurement for selected variables and scales }\end{array}$ & 34 & 3 & 1998 & $\mathrm{C}$ \\
\hline Rehak J & $\begin{array}{l}\text { SOCIOL } \\
\text { CAS }\end{array}$ & $\begin{array}{l}\text { Quality of data I. Classical model of measuring } \\
\text { reliability and its practical application }\end{array}$ & 34 & 1 & 1998 & $\mathrm{C}$ \\
\hline Rehakova B & $\begin{array}{l}\text { SOCIOL } \\
\text { CAS }\end{array}$ & $\begin{array}{l}\text { Transition to university under communism and } \\
\text { after its demise - The role of socio-economic } \\
\text { background in the transition between secondary } \\
\text { and tertiary education in the Czech Republic } \\
1948-1998\end{array}$ & 39 & 3 & 2003 & $E$ \\
\hline Rehakova B & $\begin{array}{l}\text { SOCIOLOGI } \\
\text { A }\end{array}$ & $\begin{array}{l}\text { Cultural and socio-economic origins of unequal } \\
\text { opportunities in attaining higher education in the } \\
\text { Czech Republic (1948-1999). }\end{array}$ & 36 & 1 & 2004 & $\mathrm{~S}$ \\
\hline Rehakova B & $\begin{array}{l}\text { SOCIOL } \\
\text { CAS }\end{array}$ & $\begin{array}{l}\text { Measuring value orientations with the use of S. H. } \\
\text { Schwartzs value portraits }\end{array}$ & 42 & 1 & 2006 & $\mathrm{C}$ \\
\hline Rehakova B & \begin{tabular}{|l|} 
SOCIOL \\
CAS \\
\end{tabular} & $\begin{array}{l}\text { The nation, national identity, and national pride in } \\
\text { Europe }\end{array}$ & 40 & 4 & 2004 & $\mathrm{C}$ \\
\hline Rehakova B & $\begin{array}{l}\text { SOCIOL } \\
\text { CAS }\end{array}$ & Friendship patterns in Czech society & 39 & 4 & 2003 & $\mathrm{C}$ \\
\hline Rehakova B & $\begin{array}{l}\text { SOCIOL } \\
\text { CAS }\end{array}$ & $\begin{array}{l}\text { Determinants in the relationship to the protection } \\
\text { of the environment }\end{array}$ & 37 & 4 & 2001 & $\mathrm{C}$ \\
\hline Rehakova B & $\begin{array}{l}\text { SOCIOL } \\
\text { CAS }\end{array}$ & Introducing logistic regression & 36 & 4 & 2000 & $\mathrm{C}$ \\
\hline Rehakova B & $\begin{array}{l}\text { SOCIOL } \\
\text { CAS }\end{array}$ & Perceived and desired income inequalities & 36 & 1 & 2000 & $\mathrm{C}$ \\
\hline Rehakova B & $\begin{array}{l}\text { SOCIOL } \\
\text { CAS }\end{array}$ & $\begin{array}{l}\text { The } 1998 \text { early elections: The voting behaviour of } \\
\text { various groups of voters }\end{array}$ & 35 & 3 & 1999 & $\mathrm{C}$ \\
\hline Rehakova B & $\begin{array}{l}\text { SOCIOL } \\
\text { CAS }\end{array}$ & The development of beliefs about just income & 34 & 1 & 1998 & $\mathrm{C}$ \\
\hline Rysavy D & $\begin{array}{l}\text { SOCIOL } \\
\text { CAS }\end{array}$ & $\begin{array}{l}\text { Municipal is municipal and big is big! A hypothesis } \\
\text { about the politicisation of local political elites }\end{array}$ & 42 & 5 & 2006 & $\mathrm{C}$ \\
\hline
\end{tabular}




\begin{tabular}{|c|c|c|c|c|c|c|}
\hline Rysavy D & \begin{tabular}{|l|} 
SOCIOL \\
CAS \\
\end{tabular} & $\begin{array}{l}\text { Social distance towards the Roma. The case of } \\
\text { university students }\end{array}$ & 39 & 1 & 2003 & $\mathrm{C}$ \\
\hline Rysavy D & $\begin{array}{l}\text { SOCIOL } \\
\text { CAS }\end{array}$ & $\begin{array}{l}\text { Foreign and/versus native scholar - Toward } \\
\text { methodology of Transitional processes project }\end{array}$ & 38 & $\begin{array}{l}1 \\
2\end{array}$ & 2002 & $\mathrm{C}$ \\
\hline $\begin{array}{l}\text { Salamounova } \\
\text { P }\end{array}$ & $\begin{array}{l}\text { SOCIOL } \\
\text { CAS }\end{array}$ & $\begin{array}{l}\text { Attitudes towards immigrants and the impact of } \\
\text { migration in European countries }\end{array}$ & 42 & 1 & 2006 & $\mathrm{C}$ \\
\hline Sanderova J & $\begin{array}{l}\text { SOCIOL } \\
\text { CAS }\end{array}$ & $\begin{array}{l}\text { Classes and strata in contemporary capitalism } \\
\text { again }\end{array}$ & 35 & 1 & 1999 & $\mathrm{C}$ \\
\hline Simonova N & $\begin{array}{l}\text { SOCIOL } \\
\text { CAS }\end{array}$ & $\begin{array}{l}\text { Czech and Polish higher education - from } \\
\text { bureaucracy to market competition }\end{array}$ & 42 & 3 & 2006 & $\mathrm{C}$ \\
\hline Simonova N & $\begin{array}{l}\text { SOCIOL } \\
\text { CAS }\end{array}$ & $\begin{array}{l}\text { Transition to university under communism and } \\
\text { after its demise - The role of socio-economic } \\
\text { background in the transition between secondary } \\
\text { and tertiary education in the Czech Republic } \\
1948-1998\end{array}$ & 39 & 3 & 2003 & $\mathrm{E}$ \\
\hline Simonova N & $\begin{array}{l}\text { SOCIOL } \\
\text { CAS }\end{array}$ & Czech higher education still at the crossroads & 39 & 3 & 2003 & $E$ \\
\hline Simonova N & $\begin{array}{l}\text { SOCIOLOGI } \\
\text { A }\end{array}$ & $\begin{array}{l}\text { Cultural and socio-economic origins of unequal } \\
\text { opportunities in attaining higher education in the } \\
\text { Czech Republic (1948-1999). }\end{array}$ & 36 & 1 & 2004 & $\mathrm{~S}$ \\
\hline Simonova N & $\begin{array}{l}\text { SOCIOL } \\
\text { CAS }\end{array}$ & $\begin{array}{l}\text { Czech and Polish higher education - from } \\
\text { bureaucracy to market competition }\end{array}$ & 42 & 3 & 2006 & $\mathrm{E}$ \\
\hline Simonova N & $\begin{array}{l}\text { BRIT J } \\
\text { SOCIOL } \\
\text { EDUC }\end{array}$ & $\begin{array}{l}\text { The evolution of educational inequalities in the } \\
\text { Czech Republic after } 1989\end{array}$ & 24 & 4 & 2003 & $\mathrm{E}$ \\
\hline Sirovatka T & $\begin{array}{l}\text { SOCIOL } \\
\text { CAS }\end{array}$ & $\begin{array}{l}\text { Poverty, deprivation and social exlusion: The } \\
\text { unemployed and the working poor }\end{array}$ & 42 & 4 & 2006 & $\mathrm{C}$ \\
\hline Sirovatka T & $\begin{array}{l}\text { SOC } \\
\text { POLICY } \\
\text { ADMIN }\end{array}$ & $\begin{array}{l}\text { Poverty, social exclusion and social policy in the } \\
\text { Czech Republic }\end{array}$ & 40 & 3 & 2006 & $\mathrm{E}$ \\
\hline Sirovatka T & \begin{tabular}{|l|} 
FINANC \\
UVER
\end{tabular} & $\begin{array}{l}\text { Unemployment, labour marginalisation and } \\
\text { deprivation }\end{array}$ & 55 & $\begin{array}{l}1 \\
2\end{array}$ & 2005 & $\mathrm{E}$ \\
\hline Sirovatka T & $\begin{array}{l}\text { SOCIOL } \\
\text { CAS }\end{array}$ & $\begin{array}{l}\text { The long-term unemployed - Living conditions and } \\
\text { strategies }\end{array}$ & 39 & 1 & 2003 & $\mathrm{C}$ \\
\hline Sirovatka T & $\begin{array}{l}\text { SOCIOL } \\
\text { CAS }\end{array}$ & The Czech welfare state and its legitimacy & 35 & 4 & 1999 & $\mathrm{C}$ \\
\hline Sirovatka T & $\begin{array}{l}\text { SOCIOL } \\
\text { CAS }\end{array}$ & $\begin{array}{l}\text { Welfare state solidarity and support. The Czech } \\
\text { Republic and the Netherlands in comparison }\end{array}$ & 35 & 3 & 1999 & $\mathrm{C}$ \\
\hline Sirovatka T & POLIT EKON & Unemployment and work incentives & 51 & 3 & 2003 & $\mathrm{C}$ \\
\hline Sirovatka T & $\begin{array}{l}\text { SOCIOL } \\
\text { CAS }\end{array}$ & Opinions of Czechs about the welfare state & 38 & 3 & 2002 & $E$ \\
\hline Sirovatka T & POLIT EKON & $\begin{array}{l}\text { The Czech labor market in the second half of the } \\
\text { nineties }\end{array}$ & 50 & 3 & 2002 & $\mathrm{C}$ \\
\hline Sirovatka T & $\begin{array}{l}\text { FINANC } \\
\text { UVER }\end{array}$ & $\begin{array}{l}\text { Redistribution legitimacy: Czech Republic in } \\
\text { international comparison }\end{array}$ & 52 & 1 & 2002 & $\mathrm{C}$ \\
\hline Sirovatka T & \begin{tabular}{|l|} 
EASTERN \\
EUR ECON
\end{tabular} & $\begin{array}{l}\text { Social transfers and their effect in the } \\
\text { transformation period }\end{array}$ & 38 & 6 & 2000 & $\mathrm{E}$ \\
\hline Sirovatka T & $\begin{array}{l}\text { SOCIOL } \\
\text { CAS }\end{array}$ & $\begin{array}{l}\text { Welfare benefits recipients: On a "social } \\
\text { springboard" or at the dead end? }\end{array}$ & 36 & 2 & 2000 & $\mathrm{C}$ \\
\hline Sirovatka T & POLIT EKON & Effectiveness of the Czech social policy & 47 & 6 & 1999 & $\mathrm{C}$ \\
\hline Sirovatka T & POLIT EKON & $\begin{array}{l}\text { Social transfers in the transformation period and } \\
\text { their effects }\end{array}$ & 46 & 4 & 1998 & $\mathrm{C}$ \\
\hline Skalnik P & $\begin{array}{l}\text { SOCIOL } \\
\text { CAS }\end{array}$ & $\begin{array}{l}\text { Politics of social anthropology on the Czech } \\
\text { academic scene after } 1989 \text { - Report by an } \\
\text { observing participant }\end{array}$ & 38 & $\begin{array}{l}1 \\
2\end{array}$ & \begin{tabular}{|l|}
2002 \\
\end{tabular} & $\mathrm{C}$ \\
\hline Skovajsa M & $\begin{array}{l}\text { POL SOCIOL } \\
\text { REV }\end{array}$ & $\begin{array}{l}\text { Non-profit foundations in four countries of central } \\
\text { and eastern Europe }\end{array}$ & & $\begin{array}{c}15 \\
4\end{array}$ & 2006 & $E$ \\
\hline
\end{tabular}




\begin{tabular}{|c|c|c|c|c|c|c|}
\hline Skovajsa M & $\begin{array}{l}\text { SOCIOL } \\
\text { CAS }\end{array}$ & $\begin{array}{l}\text { A sociologist fascinated by the singular: The } \\
\text { epistemology of Raymond Aron }\end{array}$ & 41 & 5 & 2005 & $\mathrm{C}$ \\
\hline Skovajsa M & FILOS CAS & Gadamer centenarius & 48 & 3 & 2000 & $\mathrm{C}$ \\
\hline Slepickova L & $\begin{array}{l}\text { SOCIOL } \\
\text { CAS }\end{array}$ & $\begin{array}{l}\text { Involuntary childlessness in a sociological } \\
\text { perspective }\end{array}$ & 42 & 5 & 2006 & $\mathrm{C}$ \\
\hline Smith ML & $\begin{array}{l}\text { SOCIOL } \\
\text { CAS }\end{array}$ & $\begin{array}{l}\text { Determination of college expectations in OECD } \\
\text { countries: The role of individual and structural } \\
\text { factors }\end{array}$ & 43 & 6 & 2007 & $\mathrm{E}$ \\
\hline Sokol J & \begin{tabular}{|l|} 
SOCIOL \\
CAS \\
\end{tabular} & What is money? & 40 & 4 & 2004 & $\mathrm{C}$ \\
\hline Sokol J & $\begin{array}{l}\text { OSTEUROP } \\
\text { A } \\
\end{array}$ & $\begin{array}{l}\text { Linguistic diversity in Europe and its political } \\
\text { significance }\end{array}$ & 54 & $\begin{array}{l}5, \\
6 \\
\end{array}$ & 2004 & $\mathrm{G}$ \\
\hline Sokol J & PHIL TODAY & $\begin{array}{l}\text { An address from elsewhere - (The message of } \\
\text { Levinas in the Czech dissident milieu) }\end{array}$ & 43 & 2 & 1999 & $\mathrm{E}$ \\
\hline Soukup P & \begin{tabular}{|l|} 
SOCIOL \\
CAS \\
\end{tabular} & Why use hierarchical linear models? & 42 & 5 & 2006 & $\mathrm{C}$ \\
\hline Soukup P & $\begin{array}{l}\text { SOCIOL } \\
\text { CAS }\end{array}$ & $\begin{array}{l}\text { Some notes on the obsession of the Czech social } \\
\text { sciences with statistical significance }\end{array}$ & 43 & 2 & 2007 & $\mathrm{C}$ \\
\hline Soukup P & $\begin{array}{l}\text { SOCIOL } \\
\text { CAS }\end{array}$ & $\begin{array}{l}\text { Determination of college expectations in OECD } \\
\text { countries: The role of individual and structural } \\
\text { factors }\end{array}$ & 43 & 6 & 2007 & $E$ \\
\hline Stachova J & $\begin{array}{l}\text { SOCIOL } \\
\text { CAS }\end{array}$ & $\begin{array}{l}\text { The regional dimension of the political culture of } \\
\text { the Czech population }\end{array}$ & 41 & 5 & 2005 & C \\
\hline Stastna A & $\begin{array}{l}\text { SOCIOL } \\
\text { CAS }\end{array}$ & $\begin{array}{l}\text { A second child in the family - The preferences and } \\
\text { values of Czech women }\end{array}$ & 43 & 4 & 2007 & $\mathrm{C}$ \\
\hline Strakova J & $\begin{array}{l}\text { BRIT J } \\
\text { SOCIOL } \\
\text { EDUC }\end{array}$ & $\begin{array}{l}\text { The role of the family and the school in the } \\
\text { reproduction of educational inequalities in the } \\
\text { post-Communist Czech Republic }\end{array}$ & 26 & 1 & 2005 & $E$ \\
\hline Strakova J & $\begin{array}{l}\text { SOCIOL } \\
\text { CAS }\end{array}$ & $\begin{array}{l}\text { The role of the family and the school in the } \\
\text { reproduction of educational inequalities - A } \\
\text { sociological look at the role of multi-year gymnasia } \\
\text { in the context of the results of the PISA } 2000 \\
\text { study }\end{array}$ & 39 & 5 & 2003 & $\mathrm{C}$ \\
\hline Strakova J & $\begin{array}{l}\text { SOCIOL } \\
\text { CAS }\end{array}$ & $\begin{array}{l}\text { Gender differences in the Czech Republic from } \\
\text { the perspective of international large-scale } \\
\text { surveys }\end{array}$ & 42 & 4 & 2006 & $\mathrm{C}$ \\
\hline Strakova J & $\begin{array}{l}\text { SOCIOL } \\
\text { CAS }\end{array}$ & $\begin{array}{l}\text { International large-scale studies of educational } \\
\text { achievement - The involvement of the Czech } \\
\text { Republic }\end{array}$ & 39 & 3 & 2003 & $E$ \\
\hline Strakova J & $\begin{array}{l}\text { SOCIOL } \\
\text { CAS }\end{array}$ & $\begin{array}{l}\text { The impact of the structure of the education } \\
\text { system on the development of educational } \\
\text { inequalities in the Czech Republic }\end{array}$ & 43 & 3 & 2007 & $E$ \\
\hline Subrt J & $\begin{array}{l}\text { SOCIOL } \\
\text { CAS }\end{array}$ & $\begin{array}{l}\text { The theatrical approach of Erving Goffman - On } \\
\text { the Czech publication of Goffman's book The } \\
\text { presentation of self in everyday life }\end{array}$ & 37 & 2 & 2001 & $\mathrm{C}$ \\
\hline Subrt J & $\begin{array}{l}\text { SOCIOL } \\
\text { CAS }\end{array}$ & Time and discipline: The monastery as a model & 35 & 1 & 1999 & $\mathrm{C}$ \\
\hline Sunega $\mathrm{P}$ & $\begin{array}{l}\text { SOCIOL } \\
\text { CAS }\end{array}$ & $\begin{array}{l}\text { Housing affordability of rental and owner-occupied } \\
\text { housing over the course of the economic } \\
\text { transformation in the Czech Republic (1991-2003) }\end{array}$ & 42 & 5 & 2006 & $\mathrm{C}$ \\
\hline Sunega $\mathrm{P}$ & \begin{tabular}{|l|} 
FINANC \\
UVER \\
\end{tabular} & Equilibrium rent and housing policy implications & 53 & $\begin{array}{l}1 \\
2\end{array}$ & 2003 & $\mathrm{C}$ \\
\hline Sunega $P$ & $\begin{array}{l}\text { SOCIOL } \\
\text { CAS }\end{array}$ & $\begin{array}{l}\text { The effectiveness of selected housing policy } \\
\text { subsidies in the Czech Republic }\end{array}$ & 41 & 2 & 2005 & $\mathrm{C}$ \\
\hline Sunega $P$ & $\begin{array}{l}\text { SOCIOL } \\
\text { CAS }\end{array}$ & $\begin{array}{l}\text { The effect of housing conditions on the intended } \\
\text { labour migration of the Czech population }\end{array}$ & 43 & 2 & 2007 & $\mathrm{C}$ \\
\hline Sykorova D & $\begin{array}{l}\text { SOCIOL } \\
\text { CAS }\end{array}$ & $\begin{array}{l}\text { From solidarity as a basis of intergenerational. } \\
\text { Family relations to ambivalence and negotiating }\end{array}$ & 42 & 4 & 2006 & $\mathrm{C}$ \\
\hline
\end{tabular}




\begin{tabular}{|c|c|c|c|c|c|c|}
\hline Sykorova D & \begin{tabular}{|l} 
SOCIOLOGI \\
A
\end{tabular} & $\begin{array}{l}\text { Seniors in the Czech Republic. Myths and their } \\
\text { deconstruction. }\end{array}$ & 38 & 2 & 2006 & $\mathrm{~S}$ \\
\hline Sykorova D & $\begin{array}{l}\text { SOCIOL } \\
\text { CAS }\end{array}$ & $\begin{array}{l}\text { The family and community in central Europe - } \\
\text { Information from the survey }\end{array}$ & 35 & 2 & 1999 & $\mathrm{C}$ \\
\hline Szalo C & $\begin{array}{l}\text { SOCIOL } \\
\text { CAS }\end{array}$ & $\begin{array}{l}\text { Eight women migrants and their shared } \\
\text { transnational world }\end{array}$ & 43 & 1 & 2007 & $\mathrm{~S}$ \\
\hline Trnka R & $\begin{array}{l}\text { PERCEPT } \\
\text { MOTOR } \\
\text { SKILL }\end{array}$ & $\begin{array}{l}\text { Sex of expresser and correct perception of facial } \\
\text { expressions of emotion }\end{array}$ & 104 & 3 & 2007 & $E$ \\
\hline Tucek M & $\begin{array}{l}\text { SOTSIOL } \\
\text { ISSLED }\end{array}$ & $\begin{array}{l}\text { Transformation and modernization of Czech } \\
\text { society }\end{array}$ & $?$ & 7 & 2002 & $\mathrm{R}$ \\
\hline Tucek M & $\begin{array}{l}\text { SOCIOL } \\
\text { CAS }\end{array}$ & $\begin{array}{l}\text { The Czech economic elite after fifteen years of } \\
\text { post-socialist transformation }\end{array}$ & 42 & 3 & 2006 & $E$ \\
\hline Vail BJ & $\begin{array}{l}\text { SOCIOL } \\
\text { CAS }\end{array}$ & $\begin{array}{l}\text { Illegal waste transport and the Czech Republic: } \\
\text { An environmental sociological perspective }\end{array}$ & 43 & 6 & 2007 & $E$ \\
\hline Vajdova Z & $\begin{array}{l}\text { SOCIOL } \\
\text { CAS }\end{array}$ & $\begin{array}{l}\text { The regional dimension of the political culture of } \\
\text { the Czech population }\end{array}$ & 41 & 5 & 2005 & $\mathrm{C}$ \\
\hline Vajdova Z & $\begin{array}{l}\text { SOCIOL } \\
\text { CAS }\end{array}$ & $\begin{array}{l}\text { Housing paths realised through the market - } \\
\text { Information from the survey }\end{array}$ & 36 & 1 & 2000 & $\mathrm{C}$ \\
\hline Valentova M & $\begin{array}{l}\text { FINANC } \\
\text { UVER }\end{array}$ & $\begin{array}{l}\text { Redistribution legitimacy: Czech Republic in } \\
\text { international comparison }\end{array}$ & 52 & 1 & 2002 & $\mathrm{C}$ \\
\hline Vasecka M & $\begin{array}{l}\text { SOCIOLOGI } \\
\text { A }\end{array}$ & $\begin{array}{l}\text { Research of antisemitism in Slovakia after } 1989 \text { in } \\
\text { a critical perspective. }\end{array}$ & 38 & 4 & 2006 & $\mathrm{~S}$ \\
\hline Vecernik J & $\begin{array}{l}\text { SOCIOL } \\
\text { CAS }\end{array}$ & $\begin{array}{l}\text { Work values and job attitudes in the Czech } \\
\text { Republic between } 1997 \text { and } 2005\end{array}$ & 42 & 6 & 2006 & $E$ \\
\hline Vecernik J & $\begin{array}{l}\text { FINANC } \\
\text { UVER }\end{array}$ & $\begin{array}{l}\text { Income taxes and benefits among Czech } \\
\text { employees: Changes since } 1989 \text { and a cross- } \\
\text { national comparison }\end{array}$ & 56 & $\begin{array}{l}1 \\
2\end{array}$ & 2006 & $E$ \\
\hline Vecernik J & $\begin{array}{l}\text { SOCIOL } \\
\text { CAS }\end{array}$ & Changes and issues in Czech social policy & 41 & 5 & 2005 & $\mathrm{C}$ \\
\hline Vecernik J & $\begin{array}{l}\text { FINANC } \\
\text { UVER }\end{array}$ & $\begin{array}{l}\text { The labour market in the CR: Trends, policies and } \\
\text { attitudes }\end{array}$ & 55 & $\begin{array}{l}1, \\
2\end{array}$ & 2005 & $E$ \\
\hline Vecernik J & $\begin{array}{l}\text { SOCIOL } \\
\text { CAS }\end{array}$ & $\begin{array}{l}\text { Who is poor in the Czech Republic? The changing } \\
\text { structure and faces of poverty after } 1989\end{array}$ & 40 & 6 & 2004 & $E$ \\
\hline Vecernik J & $\begin{array}{l}\text { SOCIOL } \\
\text { CAS }\end{array}$ & $\begin{array}{l}\text { Monitoring the transition in the Czech Republic: } \\
\text { Data, surveys and studies }\end{array}$ & 38 & 3 & 2002 & $E$ \\
\hline Vecernik J & $\begin{array}{l}\text { SOCIOL } \\
\text { CAS }\end{array}$ & Transition studies and Czech sociology & 38 & $\begin{array}{l}1, \\
2\end{array}$ & 2002 & $\mathrm{C}$ \\
\hline Vecernik J & $\begin{array}{l}\text { SOCIOL } \\
\text { EDUC }\end{array}$ & Education and support for the Czech reforms & 75 & 2 & 2002 & $E$ \\
\hline Vecernik J & $\begin{array}{l}\text { FINANC } \\
\text { UVER }\end{array}$ & $\begin{array}{l}\text { Income redistribution via taxes and benefits in the } \\
\text { CR: Change and perception after } 1989\end{array}$ & 52 & 1 & 2002 & $\mathrm{C}$ \\
\hline Vecernik J & $\begin{array}{l}\text { J POLIT MIL } \\
\text { SOCIOL }\end{array}$ & $\begin{array}{l}\text { Trends in Czech attitudes toward the market and } \\
\text { democracy }\end{array}$ & 29 & 2 & 2001 & $\mathrm{E}$ \\
\hline Vecernik J & \begin{tabular}{|l|}
$\mathrm{SOClOL}$ \\
$\mathrm{CAS}$
\end{tabular} & Social reporting in the Czech Republic since 1989 & 37 & 3 & 2001 & $\mathrm{C}$ \\
\hline Vecernik J & \begin{tabular}{|l|} 
SOCIOL \\
CAS \\
\end{tabular} & $\begin{array}{l}\text { Age and Czechs' attitudes about the post- } \\
\text { communist reforms }\end{array}$ & 37 & 3 & 2001 & $\mathrm{C}$ \\
\hline Vecernik J & $\begin{array}{l}\text { FINANC } \\
\text { UVER }\end{array}$ & $\begin{array}{l}\text { Earnings disparities in the CR: Evidence from the } \\
\text { nineties and a cross-national comparison }\end{array}$ & 51 & 9 & 2001 & $\mathrm{C}$ \\
\hline Vecernik J & $\begin{array}{l}\text { SOCIOL } \\
\text { QUART }\end{array}$ & $\begin{array}{l}\text { Age and Czechs' attitudes toward the } \\
\text { postcommunist economic reforms }\end{array}$ & 42 & 3 & 2001 & $\mathrm{E}$ \\
\hline Vecernik J & $\begin{array}{l}\text { SOCIOL } \\
\text { CAS }\end{array}$ & $\begin{array}{l}\text { Education, economic experiences, anxiety about } \\
\text { and support for the Czech reforms }\end{array}$ & 36 & 4 & 2000 & $\mathrm{C}$ \\
\hline Vecernik J & POLIT EKON & $\begin{array}{l}\text { Social problems and policies in the Czech republic } \\
\text { after 1989: Transition costs and social structure }\end{array}$ & 48 & 4 & 2000 & $\mathrm{E}$ \\
\hline
\end{tabular}




\begin{tabular}{|c|c|c|c|c|c|c|}
\hline Vecernik J & $\begin{array}{l}\text { FINANC } \\
\text { UVER }\end{array}$ & $\begin{array}{l}\text { Income distribution in the Czech Republic, 1988- } \\
\text { 1996: A market economy reborn }\end{array}$ & 49 & 8 & 1999 & $\mathrm{C}$ \\
\hline Vecernik J & $\begin{array}{l}\text { COMMUNIS } \\
\text { POST- } \\
\text { COMMUN }\end{array}$ & $\begin{array}{l}\text { The middle class in the Czech reforms: the } \\
\text { interplay between policies and social stratification }\end{array}$ & 32 & 4 & 1999 & $E$ \\
\hline Vecernik J & $\begin{array}{l}\text { RURAL } \\
\text { SOCIOL }\end{array}$ & $\begin{array}{l}\text { Rural and urban differences in economic } \\
\text { experiences, anxiety and support for the post- } \\
\text { communist reforms in the Czech and Slovak } \\
\text { Republics }\end{array}$ & 64 & 3 & 1999 & $\mathrm{E}$ \\
\hline Vecernik J & $\begin{array}{l}\text { SOCIOL } \\
\text { CAS }\end{array}$ & $\begin{array}{l}\text { The middle classes in Czech reforms - The } \\
\text { interplay between socio-economic policies and } \\
\text { social stratification }\end{array}$ & 35 & 1 & 1999 & $\mathrm{C}$ \\
\hline Vesely A & $\begin{array}{l}\text { BRIT J } \\
\text { PSYCHIAT }\end{array}$ & $\begin{array}{l}\text { Violent behaviour in schizophrenia - Retrospective } \\
\text { study of four independent samples from Prague, } \\
1949 \text { to } 2000\end{array}$ & 187 & & 2005 & $\mathrm{E}$ \\
\hline Vesely A & $\begin{array}{l}\text { SOCIOL } \\
\text { CAS } \\
\end{array}$ & The knowledge society as a theoretical concept & 40 & 4 & 2004 & $\mathrm{C}$ \\
\hline Vinopal J & \begin{tabular}{|l|}
$\mathrm{SOClOL}$ \\
$\mathrm{CAS}$
\end{tabular} & $\begin{array}{l}\text { The empirical accessibility of left-right political } \\
\text { orientations }\end{array}$ & 42 & 1 & 2006 & $\mathrm{C}$ \\
\hline Vinopal J & $\begin{array}{l}\text { SOCIOL } \\
\text { CAS }\end{array}$ & $\begin{array}{l}\text { Verification of indicators used in research of } \\
\text { opinions on the crime-rate }\end{array}$ & 37 & 1 & 2001 & $\mathrm{C}$ \\
\hline Vlachova K & $\begin{array}{l}\text { SOCIOL } \\
\text { CAS } \\
\end{array}$ & $\begin{array}{l}\text { The social background of the extreme right in the } \\
\text { Czech Republic }\end{array}$ & 35 & 3 & 1999 & $\mathrm{C}$ \\
\hline Vlachova K & $\begin{array}{l}\text { SOCIOL } \\
\text { CAS }\end{array}$ & $\begin{array}{l}\text { Active citizenship and satisfaction with democracy } \\
\text { in Europe }\end{array}$ & 42 & 1 & 2006 & $\mathrm{C}$ \\
\hline Vlachova K & $\begin{array}{l}\text { SOCIOL } \\
\text { CAS }\end{array}$ & $\begin{array}{l}\text { The crystallisation of political attitudes and the } \\
\text { political spectrum in the Czech Republic }\end{array}$ & 34 & 2 & 1998 & $\mathrm{C}$ \\
\hline Vlachova K & $\begin{array}{l}\text { SOCIOL } \\
\text { CAS }\end{array}$ & $\begin{array}{l}\text { The role of politically relevant attitudes and value } \\
\text { orientations in electoral decisions. The Czech } \\
\text { Republic in } 1996\end{array}$ & 34 & 2 & 1998 & $\mathrm{C}$ \\
\hline Vlachova K & $\begin{array}{l}\text { COMMUNIS } \\
\text { POST- } \\
\text { COMMUN }\end{array}$ & $\begin{array}{l}\text { Values and electoral decisions in the Czech } \\
\text { Republic }\end{array}$ & 31 & 3 & 1998 & $E$ \\
\hline Vlachova K & $\begin{array}{l}\text { SOCIOL } \\
\text { CAS }\end{array}$ & $\begin{array}{l}\text { The nation, national identity, and national pride in } \\
\text { Europe }\end{array}$ & 40 & 4 & 2004 & $\mathrm{C}$ \\
\hline Vlachova K & $\begin{array}{l}\text { SOCIOL } \\
\text { CAS }\end{array}$ & $\begin{array}{l}\text { The dynamics of positive and negative-party } \\
\text { identification in the Czech Republic }\end{array}$ & 39 & 4 & 2003 & $\mathrm{C}$ \\
\hline Vlachova K & $\begin{array}{l}\text { COMMUNIS } \\
\text { POST- } \\
\text { COMMUN }\end{array}$ & $\begin{array}{l}\text { Party identification in the Czech Republic: inter- } \\
\text { party hostility and party preference }\end{array}$ & 34 & 4 & 2001 & $E$ \\
\hline Vojtiskova K & $\begin{array}{l}\text { SOCIOL } \\
\text { CAS }\end{array}$ & $\begin{array}{l}\text { The 'Background' and 'Battlefield' in the struggle } \\
\text { for justice: Text analysis of trade union, feminist, } \\
\text { and human rights media communications }\end{array}$ & 42 & 2 & 2006 & $\mathrm{C}$ \\
\hline Vyhlidal J & $\begin{array}{l}\text { SOCIOL } \\
\text { CAS }\end{array}$ & $\begin{array}{l}\text { The long-term unemployed - Living conditions and } \\
\text { strategies }\end{array}$ & 39 & 1 & 2003 & $\mathrm{C}$ \\
\hline Zich F & $\begin{array}{l}\text { SOCIOL } \\
\text { CAS }\end{array}$ & $\begin{array}{l}\text { The problems behind the creation of a cross- } \\
\text { border society on the Czech-Germany border }\end{array}$ & 37 & 2 & 2001 & $\mathrm{C}$ \\
\hline Zizlavsky M & POLIT EKON & Unemployment and work incentives & 51 & 3 & 2003 & $\mathrm{C}$ \\
\hline Zizlavsky M & POLIT EKON & $\begin{array}{l}\text { The Czech labor market in the second half of the } \\
\text { nineties }\end{array}$ & 50 & 3 & 2002 & $\mathrm{C}$ \\
\hline
\end{tabular}


Individual researchers, as well as the on-line and printed versions of the CERGE-EI Working Papers (including their dissemination) were supported from the European Structural Fund (within the Operational Programme Prague Adaptability), the budget of the City of Prague, the Czech Republic's state budget and the following institutional grants:

- Center of Advanced Political Economy Research [Centrum pro pokročilá politickoekonomická studia], No. LC542, (2005-2009),

- Economic Aspects of EU and EMU Entry [Ekonomické aspekty vstupu do Evropské unie a Evropské měnové unie], No. AVOZ70850503, (2005-2010);

- Economic Impact of European Integration on the Czech Republic [Ekonomické dopady evropské integrace na ČR], No. MSM0021620846, (2005-2011);

Specific research support and/or other grants the researchers/publications benefited from are acknowledged at the beginning of the Paper.

(c) Josef Basl, Daniel Münich, Oleg Sidorkin, 2009

All rights reserved. No part of this publication may be reproduced, stored in a retrieval system or transmitted in any form or by any means, electronic, mechanical or photocopying, recording, or otherwise without the prior permission of the publisher.

Published by

Charles University in Prague, Center for Economic Research and Graduate Education (CERGE) and

Economics Institute ASCR, v. v. i. (EI)

CERGE-El, Politických vězňů 7, 11121 Prague 1, tel.: +420 224005 153, Czech Republic.

Printed by CERGE-EI, Prague

Subscription: CERGE-EI homepage: http://www.cerge-ei.cz

Editors: Directors of CERGE and EI

Managing editors: Deputy Directors for Research of CERGE and EI

ISSN 1211-3298

ISBN 978-80-7343-194-5 (Univerzita Karlova. Centrum pro ekonomický výzkum

a doktorské studium)

ISBN 978-80-7344-183-8 (Národohospodářský ústav AV ČR, v. v. i.) 
CERGE-EI

P.O.BOX 882

Politických vězňů 7

11121 Praha 1

Czech Republic http://www.cerge-ei.cz 